\title{
Die Bildungsexpansion in der Schweiz und ihre Folgen
}

\author{
Eine empirische Analyse des Wandels der \\ Bildungsbeteiligung und Bildungsungleichheiten \\ mit den Daten der Schweizer Volkszählungen 1970, \\ 1980, 1990 und 2000
}

\section{Rolf Becker $\cdot$ Christoph Zangger}

\begin{abstract}
Zusammenfassung: Im vorliegenden Beitrag wird für die Schweiz im 20. Jahrhundert der Zusammenhang von Bildungsexpansion und sozialer Ungleichheit von Bildungschancen nach sozialer Herkunft und Geschlecht untersucht. Im Vordergrund steht die Frage, ob für die Schweiz die These dauerhafter Bildungsungleichheit von Shavit und Blossfeld aufrechterhalten werden kann. Für die empirische Analyse werden Daten der Volkszählungen in den Jahren 1970, 1980, 1990 und 2000 herangezogen. Es kann belegt werden, dass eine moderate Bildungsexpansion und auch ein Abbau von Bildungsungleichheiten nach sozialer Herkunft und Geschlecht in der Abfolge von Geburtskohorten erfolgte. Nach 1990 bis zum Jahre 2000 jedoch haben die sozialen Ungleichheiten beim Erwerb der Studienberechtigung wieder zugenommen. Insgesamt sind sie jedoch zugunsten bislang benachteiligter Sozialschichten geringer als zu Beginn der Bildungsexpansion, sodass für die Schweiz die These dauerhafter Bildungsungleichheiten nicht weiter aufrechterhalten werden kann.
\end{abstract}

Schlüsselwörter: Bildungsexpansion · Bildungsungleichheit · Bildungschancen · Schweiz · Volkszählung · Geburtskohorten

\section{Educational expansion in Switzerland and its consequences - An empirical analysis of change in education and inequality of educational opportunities by utilizing Swiss census data 1970, 1980, 1990, and 2000}

Abstract: In this contribution the correlation of educational expansion and social inequality of educational opportunities (IEO) for social classes and gender has been investigated for Switzerland in the twentieth century. The empirical analysis is focused on the thesis of persistent

(C) Springer Fachmedien Wiesbaden 2013

R. Becker $(\bowtie) \cdot$ C. Zangger

Institut für Erziehungswissenschaft, Universität Bern,

Muesmattstr. 27, 3012 Bern, Schweiz

E-Mail: rolf.becker@edu.unibe.ch

C. Zangger

E-Mail: christoph.zangger@edu.unibe.ch 
inequalities claimed by Shavit and Blossfeld. For testing this thesis, data of the Swiss census in 1970, 1980, 1990, and 2000 has been utilized. First of all, it is found for Switzerland that the moderate educational expansion has been carried out by the succession of birth cohorts. Secondly, this process has led to decreasing IEO. However, after 1990, for the individuals younger than 22 years we have witnessed an increase of social inequalities in becoming eligible for university training. Overall, in favour of disadvantaged social classes, there is a decrease in IEO since the start of educational expansion in the 1950s. Therefore, the thesis of persistent inequalities has been not confirmed for Switzerland.

Keywords: Educational expansion - Inequality of educational opportunities - Switzerland · Census $\cdot$ Birth cohorts

\section{Einleitung}

Für das ausgehende 19. und das gesamte 20. Jahrhundert betrachtet, ist die Bildungsexpansion ein universelles Phänomen und eine der bedeutendsten gesellschaftlichen Entwicklungen (vgl. Müller 1998). In allen Sozialschichten gestiegene und längere Bildungsbeteiligung sowie graduelle Anstiege des Bildungsstandes in der Bevölkerung sind zentrale Strukturmerkmale der Bildungsexpansion (vgl. Müller et al. 1997). Diese Entwicklung verlief für viele Länder unterschiedlich, weist aber auch Gemeinsamkeiten für moderne Gesellschaften auf (vgl. Müller und Kogan 2010). In mancherlei Hinsicht belegt sie die Sonderstellung der Schweiz. So zeigen Blossfeld und Shavit (1993) in ihrer international und historisch vergleichenden Studie, dass die Schweiz im Vergleich zu den anderen 12 untersuchten Ländern eine zögerliche Bildungsexpansion zu verzeichnen hatte (vgl. Buchmann und Charles 1993). Diese blieb deutlich unter dem Niveau westeuropäischer Länder (Bornschier und Aebi 1992, S. 556). Ebenso wird in dieser Studie wie auch in jüngeren Studien für die Schweiz festgestellt, dass die Bildungsexpansion zwar mit einem Niveaueffekt, einer Anhebung des Bildungsstandes, einhergegangen ist, aber nicht mit einem Struktureffekt, sprich: keinen Abbau von vergleichsweise großen Bildungsungleichheiten mit sich gebracht hat (vgl. Lamprecht und Stamm 1996; Stamm und Lamprecht 2005). Während Buchmann und Charles (1993) sowie Pfeffer (2008) davon ausgehen, dass die Bildungsungleichheiten in der Schweiz unverändert hoch sind, berichten Hadjar und Berger (2010) mit Daten des Schweizer Haushaltspanels sowie Stamm und Lamprecht (2005) mit Daten der Schweizer Volkszählung 2000 hingegen einen moderaten Rückgang der Bildungsungleichheiten nach Geschlecht und sozialer Herkunft, wobei die Entwicklung zwischen den sozialen Klassen zugunsten der Mittelschichten und zuungunsten der Arbeiterklasse differiert (Hadjar und Berger 2010, S. 195-196).

Ihr Befund für die Schweiz steht in Einklang mit den jüngst von Breen et al. (2009, 2010) vorgelegten Studien, in denen die Analysen von Blossfeld und Shavit (1993) mit aktuelleren Daten und ungleich größeren Stichproben repliziert werden. Weil in ihrem internationalen Vergleich die Schweiz wohl aufgrund unzureichender Datenlage nicht berücksichtigt wird, werden im vorliegenden Beitrag die harmonisierten Daten der Schweizer Volkszählungen herangezogen, um den Verlauf und die Folgen der Bildungsexpansion im 20. Jahrhundert möglichst präzise nachzuzeichnen. Folgende Fragen stehen 
hierbei im Fokus der empirischen Analyse: Wie veränderte sich in der historischen Zeit über die Abfolge von Geburtskohorten - die Verteilung erworbener Bildungsabschlüsse? In welchem Umfang haben sich hierbei Bildungsungleichheiten nach sozialer Herkunft und Geschlecht gewandelt? Kann für die Schweiz die These dauerhafter Bildungsungleichheit aufrechterhalten werden?

Der Beitrag ist wie folgt aufgebaut: Im zweiten Abschnitt werden mögliche Zusammenhänge zwischen Bildungsexpansion und sich verändernden Ungleichheiten von Bildungschancen diskutiert. Der dritte Abschnitt umfasst die Beschreibung der Volkszählungsdaten und der für die Analyse verwendeten Variablen. Im vierten Abschnitt erfolgt die Darstellung der empirischen Resultate. Abschließend werden im fünften Abschnitt die Befunde zusammengefasst und im Hinblick auf die Fragestellung diskutiert.

\section{Forschungsstand und theoretischer Hintergrund}

\subsection{Forschungsstand}

Der Studie von Blossfeld und Shavit (1993) zufolge sind in elf von dreizehn modernen Industriegesellschaften mit unterschiedlichen politischen, wirtschaftlichen, kulturellen und sozialen Ordnungen trotz zunehmender Bildungsbeteiligung unterer sozialer Schichten die von der sozialen Herkunft abhängigen Bildungschancen weitgehend unverändert geblieben. Ausnahmen waren Schweden und die Niederlande. Auch Pfeffer (2008) bestätigt diese Befunde der persistenten Ungleichheit mit aktuelleren Daten für 20 Länder in der zweiten Hälfte des 20. Jahrhunderts und stellt allenfalls für zwei Länder (Finnland, Norwegen) abnehmende Bildungsungleichheiten nach sozialer Herkunft fest. Besonders gering ist die intergenerationale Bildungsmobilität in den beiden Ländern Deutschland und Schweiz, wobei sie in der Schweiz höher ist als in Deutschland (Pfeffer 2008, S. 553). Zum gleichen Ergebnis kommen Hadjar und Berger (2010, S. 195) für die herkunftsbedingten Ungleichheiten beim Erwerb höherer Bildung (Studienberechtigung und/oder Hochschulabschluss). ${ }^{1}$ Dass in diesen beiden Ländern (abgesehen von Slowenien) die Bildungsungleichheit im internationalen Vergleich am höchsten ist, führt Pfeffer (2008, S. 554 und 555) unter institutionellem Gesichtspunkt auf das standardisierte und hochgradig stratifizierte Bildungssystem zurück, das vergleichsweise nur geringe intergenerationale Bildungsmobilität zulässt.

Während in der Zwischenzeit eine Vielzahl von international und historisch vergleichenden Studien die These einer dauerhaften Bildungsungleichheit in Frage stellt (Breen 2010, S. 368), sind Studien für die Schweiz zum Zusammenhang von Bildungsexpansion und sozialer Ungleichheit von Bildungschancen rar (Buchmann und Charles 1993, S. 177). Für zwei Geburtskohorten 1949-51 und 1959-61 kommen Buchmann und

1 Zudem belegen die internationalen Ergebnisse von PISA (Programme für International Student Assessment) regelmäßig im gleichlaufenden Muster, dass - unter den Teilnehmerstaaten - in Deutschland (vgl. Baumert und Schümer 2001) und in der Schweiz (vgl. Zahner et al. 2002) der Zusammenhang von sozialer Herkunft und Lesekompetenz am stärksten ist. In Deutschland ist dieser Konnex etwas ausgeprägter als in der Schweiz. 
Charles (1993, S. 190) für die Zeit bis Ende der 1980er Jahre zum Ergebnis, dass in der Schweiz allenfalls eine verhaltene Bildungsexpansion stattgefunden habe, in deren Verlauf keine Entkoppelung der Bildungschancen von der sozialen Herkunft erfolgt sei. ${ }^{2}$ Auch für den Zugang zu den Hochschulen und den Erwerb tertiärer Bildungsabschlüsse seien die Effekte der sozialen Herkunft weitgehend stabil geblieben (Buchmann et al. 2007, S. 347). Diese Befunde für kleine Stichproben werden von Stamm und Lamprecht (2005) mit Daten der Volkszählung 2000 für unterschiedliche Altersgruppen weitgehend bestätigt. Jedoch haben sich intergenerationale Transmissionen von Bildung und Einflüsse der Klassenlage über die Zeit hinweg zwar leicht abgeschwächt, sind aber weiterhin bedeutsam (Stamm und Lamprecht 2005, S. 30). Zu ähnlichen Befunden kommen Hadjar und Berger (2010) in einer Kohortenstudie mit Daten des Schweizer Haushaltspanels, denen zufolge beim Erwerb von Bildungsabschlüssen die Arbeiterkinder ihre Rückstände etwas und die Frauen deutlich verringern konnten. Diese Entwicklung variiert zwischen den Geburtskohorten. Hadjar und Berger (2010, S. 196) zufolge haben sich die Chancen für die aus den Arbeiterklassen stammenden Personen, höhere Bildungsabschlüsse zu erwerben, in der Nachkriegszeit verbessert. Aber für die jüngeren, zwischen 1965 und 1974 Geborenen hat die Ungleichheit wieder zugenommen. Für die nach 1954 Geborenen aus den Mittelschichten hat sie gegenüber den Personen aus der Oberschicht sukzessive abgenommen. Gleiches gilt für die Frauen im Vergleich zu den Männern. Bei den älteren Kohorten hingegen zählt die Schweiz zu den Ländern wie Belgien, Großbritannien, Norwegen, Österreich und Deutschland, in denen die Benachteiligung von Frauen im Bildungssystem besonders ausgeprägt ist (vgl. Müller et al. 1997, S. 216).

Es gibt mehrere Gründe, warum sich auch für die Schweiz unterschiedliche Befunde zum Zusammenhang von Bildungsexpansion und sozial ungleichen Bildungschancen ergeben. Zum einen werden vornehmlich Datensätze mit geringer Fallzahl verwendet (z. B. Buchmann und Charles 1993; Hadjar und Berger 2010; Pfeffer 2008). Denen mangelt es aber an der nötigen ,statistical power“", um geringfügige Veränderungen bei herkunfts- oder geschlechtsbedingten Bildungschancen über längere Zeiträume aufzudecken. Zudem variieren die historischen Zeitfenster, in denen die Entwicklung der Bildungsexpansion und ihre Folgen betrachtet werden. Offensichtlich haben die früheren Studien mit den inzwischen historischen Daten noch nicht die Struktureffekte der Bildungsexpansion in ausreichender Trennschärfe erfassen können (vgl. Breen et al. 2012; Müller 1998). Schließlich werden jeweils zentrale Variablen wie Bildung und soziale Herkunft in unterschiedlicher Weise operationalisiert. Inkompatible Operationalisierungen von Bildung oder Herkunft können zu differierenden Befunden von Bildungsungleichheiten und ihren Veränderungen über die Zeit hinweg führen (vgl. Müller und Klein 2008). Zum anderen wurden amtliche Daten nicht genügend mit angemessenen Analysedesigns, statistischen Verfahren und Modellspezifikationen ausgereizt, um den Wandel von Bildungsungleichheiten infolge der Bildungsexpansion zu beschreiben. Deswegen wird im vorliegenden Beitrag eine weiterführende Analyse mit Schweizer Volkszählungsdaten vorgenommen.

2 In der Vorkriegszeit gehörte die Schweiz zu den Vorreitern einer Bildungsexpansion, büßte dann aber nach 1945 unter den europäischen Ländern ihre Spitzenposition ein (Schneider 1982, S. 217; Bornschier und Aebi 1992, S. 539). Diesen Rückstand konnte die Schweiz offensichtlich bis heute nicht mehr wettmachen. 
2.2 Institutionelle und strukturelle Rahmenbedingungen des Schweizer Bildungssystems

Besonderheiten der strukturellen und institutionellen Rahmenbedingungen des Schweizer Bildungssystems und dessen Entwicklung im 20. Jahrhundert (vor allem in den letzten Jahrzehnten) sind ein weiterer gewichtiger Grund, um die Folgen der Bildungsexpansion für die soziale Ungleichheit von Bildungschancen nochmals im Detail mit informationsreichen Daten zu untersuchen. Zum einen hat die Schweiz aufgrund ihrer föderalen Struktur und Untergliederung in 26 Kantone mit jeweils einem eigenständigen Bildungssystem wohl das am meisten dezentralisierte Bildungssystem unter den von Breen et al. (2009, 2010, 2012) untersuchten Ländern (vgl. Heidenheimer 1997; Hega 2000, S. 1). Mit den Sprachgrenzen unterscheiden sich die Kantone darin, in welchem Grad die Bildungssysteme zentral gesteuert und finanziert werden. Er ist hoch in der Romandie und im Tessin, aber niedrig in den Kantonen der Deutschschweiz. Die nationale Regierung der Schweiz (Bundesrat) spielte in der Bildungspolitik schon immer so gut wie keine Rolle (vgl. Heidenheimer 1997), während rund 90\% der bildungspolitischen Beschlussfassungen auf der Ebene der Kantone und ihrer Gemeinden erfolgen (vgl. Hega 2000). Allenfalls für Bereiche der Berufs- und Hochschulbildung hat der Bundesrat eine geringfügige legislative und administrative Autorität. So ist der Bund der Träger der beiden ETHs und zu einem Teil für die Fachhochschulen verantwortlich, während die Kantone für die kantonalen Universitäten und Hochschulen zuständig sind (vgl. Buchmann et al. 2007).

Zum anderen weist im Vergleich zu den von Breen et al. (2009, 2010) berücksichtigten Ländern die Schweiz ein besonders stratifiziertes und segmentiertes Bildungssystem mit einem mehrgliedrigen Schulsystem, einem breiten Angebot im Berufsbildungssystem und einem relativ geschlossenen binären System der Hochschulbildung auf (vgl. Buchmann et al. 2007; Müller et al. 1997; Pfeffer 2008). Aufgrund des attraktiven Ausbildungsangebots in der dualen Berufsausbildung (vgl. Wettstein und Gonon 2009; SKBF 2011) hat sie eine starke Ablenkungswirkung auf dem direkten Weg zur Hochschulbildung. So schlägt nicht nur der größte Teil eines Geburtsjahrgangs nach der Pflichtschulzeit den Weg einer dualen Berufsausbildung ein, sondern die Berufsausbildung zieht vor allem Jugendliche aus den Arbeiter- und den unteren Mittelschichten an, die durchaus befähigt oder motiviert wären, ein Hochschulstudium anzugehen. Das trägt sicherlich dazu bei, dass in der Schweiz der Zugang zur Berufsausbildung weitaus weniger selektiv nach sozialer Herkunft ist als der direkte Übergang auf das Gymnasium und die Universitäten (vgl. Becker 2010, 2012).

Jedoch hat die obligatorische Beschulung von Kindern aller Bevölkerungsschichten sowie der Ausbau eines Berufsbildungssystems in der Vor- und Zwischenkriegszeit, wie in vielen europäischen Ländern auch in der Schweiz mit seiner ausgeprägt föderalistischen politischen Ordnung (vgl. Bornschier und Aebi 1992; Rieger 2001; Schneider 1982), sowohl die gröbsten Bildungsungleichheiten beseitigt als auch zu einer Bildungsexpansion geführt (vgl. Müller und Kogan 2010). In der Nachkriegszeit wurde bei einem Ausbau der kantonalen Bildungsverwaltungen, der regionalen Dezentralisierung von Gymnasien und ihrem flächendeckenden Ausbau in der Zeit von 1965 bis 1975 (einschließlich der Schaffung von Berufsmittelschulen und Diplommittelschulen) 
(Meylan 1996, S. 10, 13) sowie der vergleichsweise verhaltenen Ausdehnung des Hochschulbereichs bei einer forcierten Ausdifferenzierung seit den 1990er Jahren durch die Einführung von Fachhochschulen und Pädagogischen Hochschulen zunächst die ausgeprägte Stratifikation und Segmentation des Bildungssystems mit seinen vielfältigen Angeboten in der sekundären beruflichen Ausbildung (Wachstum der dualen Berufsbildung zwischen 1950 und 1970; Revisionen des Bundesgesetzes über die berufliche Bildung in den Jahren 1963 und 1978; vgl. Wettstein und Gonon 2009) beibehalten. Folglich wurde auch die bestehende soziale Ungleichheit von Bildungschancen konserviert (vgl. Buchmann und Charles 1993; Rieger 2001).

Trotz Bildungsexpansion und Reformen des Bildungssystems ist die soziale Ungleichheit von Bildungschancen in der Schweiz, und das vor allem beim Erwerb der Studienberechtigung über die gymnasiale Maturität (die vergleichbar mit dem Abitur in Deutschland ist) und des Übergangs in die Hochschulen, im internationalen Vergleich betrachtet weiterhin ausgeprägt (vgl. Buchmann und Charles 1993; Buchmann et al. 2007; Müller et al. 1997, S. 190) und die Quote der Studienberechtigten, Studienanfänger und Hochschulabsolventen unterdurchschnittlich (vgl. Becker 2010; Müller et al. 1997, S. 209-210). ${ }^{3}$ So liegt der OECD-Durchschnitt für die Netto-Studierquote derzeit bei rund $60 \%$ (vgl. SKBF 2011). Während im Jahre 1960 die Quote der gymnasialen Maturität (gemessen an der ständigen Wohnbevölkerung im Alter von 19 Jahren) noch bei 4\% und 1970 bei $7 \%$ lag, stieg sie bis 1980 auf rund $10 \%$ und bis 2010 auf rund $20 \%$ an. Seit den $1980 \mathrm{er}$ Jahren haben vor allem Frauen sich verstärkt wegen der Nachfrage nach qualifizierten Arbeitskräften in den Dienstleistungs- und Verwaltungsbereichen und der Anhebung der Zugangsvoraussetzung für die Ausbildung in diesen Berufen entschieden (SKBF 2011, S. 122). Anfang der 1990er Jahren haben sich der amtlichen Statistik zufolge die Geschlechterdisparitäten bei der Maturitätsquote (gemessen an der 19-jährigen Wohnbevölkerung) zugunsten der Frauen verändert. ${ }^{4}$

Vor allem die frühzeitig einsetzende Ablenkungswirkung des Berufsbildungssystems und der hohe Aufwand für den Erwerb der Studienberechtigung dürfte den Zugang zur tertiären Ausbildung und zum Hochschulstudium weiterhin sozial exklusiv gehal-

3 Es ist auch nicht ausgeschlossen, dass der quantitative und strukturelle Ausbau des Berufsbildungs- und Hochschulsystems zu verstärkten Bildungsanstrengungen sozial benachteiligter Gruppen angereizt hat. Der flächendeckende regionale Ausbau von Schulen in der Schweiz - vor allem der von Mittelschulen und die Aufhebung von Stadt-Land-Unterschieden in der Schulversorgung in den 1950er und 1960er Jahren (Rieger 2001, S. 51 ff.) - sowie die Lehrmittelfreiheit haben zu sinkenden Kosten und folglich abnehmenden Investitionsrisiken für die längere Ausbildung der Kinder aus unteren Sozialschichten geführt. Auch das geringe Arbeitslosigkeitsrisiko für immer besser gebildete Berufsanfänger und Beschäftigte hat möglicherweise zu steigender Bildungsmotivation in diesen sozialen Klassen beigetragen (vgl. Riphahn und Sheldon 2005).

4 Dass gegenüber dieser lebenslaufbezogenen Längsschnittbetrachtung die amtliche Statistik mit einer periodenspezifischen Querschnittbetrachtung die Maturitätsquoten verzerrt wiedergibt, ist offensichtlich, weil im jeweiligen Kalenderjahr die Altersstreuung einer jeden Geburtskohorte beim Erwerb der Studienberechtigung nicht berücksichtigt wird. 
ten haben (vgl. Becker 2010; Buchmann et al. 2007). ${ }^{5}$ Wie in Deutschland (vgl. Becker 2009; Neugebauer und Schindler 2012; Neugebauer et al. 2013) bleibt der Zugang zu den Universitäten wegen geringer Durchlässigkeit der Bildungswege versperrt, wenn „nicht spätestens im Anschluss an die Sekundarstufe I der Zutritt ins Gymnasium gelingt“ (Schumann 2011, S. 252). Somit dürfte die Ablenkungswirkung des Schweizer Bildungssystems gerade für Angehörige sozial benachteiligter Sozialschichten sehr hoch sein (vgl. Becker 2010). ${ }^{6}$ Dazu trägt wahrscheinlich nicht zuletzt eine informelle Kontingentierung des Übertritts auf das Gymnasium in der Sekundarstufe II als eine weitere Hürde auf dem „Königsweg“ zur Universität bei. Diese Übertrittquoten werden inoffiziell, d.h. in der Höhe nicht gesetzlich definiert, durch die Bildungspolitik festgelegt. Diese von den Kantonen festgelegte Zulassungsbeschränkung zum Gymnasium bewirkt sicherlich zusätzlich zur Aufnahmeprüfung, Schul- und Lehrerempfehlung sowie vorherigen Allokation in die höheren Schulen der Sekundarstufe I eine zusätzliche „Siebung“, die den Aufstiegswillen von leistungsstarken und motivierten Jugendlichen aus den unteren Sozialschichten „abkühlen“ dürfte. Nach diesem politischen Willen wurde in den letzten Jahren die Zulassungsquote - mit großen Unterschieden zwischen den Kantonen - so gesetzt, dass schweizweit im Schnitt rund 20\% eines Jahrgangs der Übergang auf das Gymnasium gelingt, und diese so die Gelegenheit haben, die gymnasiale Maturität zu erwerben (vgl. Neue Zürcher Zeitung vom 28. Februar 2012). ${ }^{7}$ Auf diese Weise könnte die Ablenkung

5 Ähnlich wie in Deutschland haben auch in der Schweiz wirtschaftspolitische Diskussionen über die Nutzung von Begabungsreserven sowie der gesellschaftspolitische Diskurs über Bildung als Bürgerrecht nichts an dieser Situation geändert. Bei einem relativen Verlust an Attraktivität seit den 1970er Jahren ist die nachobligatorische Ausbildung im dualen Berufsbildungssystem nach wie vor der modale Bildungsweg. Vor allem für leistungsschwächere Jugendliche aus unteren Sozialschichten stellt die berufliche Lehre eine Art ,safety net“ beim Übergang von der Schule in den Beruf dar (vgl. Wettstein und Gonon 2009).

6 Die Attraktivität der gymnasialen Maturität für bildungsferne Gruppen in der Schweiz könnte anders als in Deutschland (vgl. Becker und Hecken 2009a) deswegen gering sein, da der Zugang zur Berufsausbildung im Anschluss an die gymnasiale Maturität in der Schweiz eine untergeordnete Rolle spielt. Zudem ist bei der Wahl zwischen einem Universitäts- oder ETH- und Fachhochschulstudium in der Schweiz zu berücksichtigen, dass „für ein Studium an einer FH in der Regel ein Berufspraktikum von zumeist 12 Monaten nachgewiesen werden muss. Dies hat vermutlich eine Erhöhung der subjektiv bewerteten Bildungskosten zur Folge" (Schumann 2011, S. 253), die bekanntlich bei sozial benachteiligten Studienberechtigten besonders schwer wiegen (vgl. Becker und Hecken 2009b). Gleiches gilt für die Möglichkeit, mit der Berufsmaturität doch noch an die Universität oder ETH zu gelangen. Entweder wird mit einer Zusatzausbildung von 4 bis 5 Semestern die gymnasiale Matura nachgeholt oder über eine Passerelle, die den Besuch von Vorbereitungskursen und einer Ergänzungsprüfung voraussetzt, wird der Weg an die Universität ermöglicht. Schließlich ist nicht ausgeschlossen, dass die Studiengebühren für das Universitätsstudium (ca. 1000-1500 CHF pro Jahr) eine ablenkende Wirkung für Jugendliche aus ökonomisch schwächeren Elternhäusern haben, sodass sie eher vom Erwerb einer Maturität und der Absicht für ein Universitätsstudium absehen (vgl. Becker 2012; Quast et al. 2012).

7 (http:/www.nzz.ch/aktuell/startseite/du-musstda-rein-1.15284726; zuletzt zugegriffen am 22.04.2013). Bislang gibt es auch keine empirisch überprüfte Erklärung für die zwischen den Kantonen variierenden Maturitätsquoten; ebenso wenig gibt es Belege dafür, dass sie auf Unterschiede in der Nachfrage, bei den schulischen Leistungen oder Zugangsbeschränkungen beruhen (SKBF 2011, S. 123). 
von Bildungsaufsteigern vom „Königsweg“ zu den Universitäten noch weiter verstärkt worden sein. Diese zunehmende Ablenkungswirkung müsste an der gestiegenen sozialen Exklusivität beim Erwerb der gymnasialen Maturität abgelesen werden können.

\subsection{Theoretischer Hintergrund für Bildungsexpansion und -ungleichheiten im sozialen Wandel}

Welche Folgewirkungen haben die zuvor geschilderten Rahmenbedingungen des Schweizer Bildungssystems auf die soziale Ungleichheit von Bildungschancen? Für die theoretische Annahme, dass die Bildungsexpansion nicht nur mit einer graduellen Höherqualifikation in der Bevölkerung einhergeht, sondern auch abnehmende Ungleichheiten von Bildungschancen nach sozialer Herkunft oder Geschlecht zur Folge haben soll, gibt es sehr unterschiedliche Erklärungsansätze. In den 1960er und 1970er Jahren dominierten makrosoziologische Ansätze wie etwa die Modernisierungstheorie (vgl. Flora 1974; Schneider 1982) oder der konflikttheoretische Ansatz (vgl. Bourdieu und Passeron 1971; Collins 1979). Der Modernisierungstheorie zufolge hängen im gesellschaftlichen Modernisierungsprozess der Zugang zu höherer Bildung und der Erwerb höherwertiger Bildungszertifikate deswegen immer weniger von sozialer Herkunft oder vom Geschlecht $\mathrm{ab}$, weil funktionale Erfordernisse der Industrieproduktion und der aufkommenden postindustriellen Gesellschaft die Arbeitgeber zwingen, Beschäftigte ausschließlich nach Produktivitätskriterien zu rekrutieren (Treiman 1970, S.217). Weil beim zunehmenden wirtschaftlichen Konkurrenzdruck Investitionen in Bildung zu komparativen Wettbewerbsvorteilen führen, bauten Staaten, wie auch die Schweiz, in der Nachkriegszeit das höhere Bildungssystem aus. Diese zusätzlichen Bildungsgelegenheiten erleichterten den „ungenutzten Bildungsreserven“ in den unteren und mittleren Sozialschichten den Zugang zu höherer Bildung. Aus funktionalistischer Sicht müsste die damit einhergehende Ablösung der „sponsored mobility“ (privilegierte Bildungschancen wegen sozialer Herkunft ohne leistungsbezogenen Wettbewerb) durch die „,contest mobility“ (Erwerb von Bildung durch Leistung im Wettbewerb) (vgl. Turner 1960) zu einer ,bildungsbasierten Meritokratie“" und damit zum Abbau herkunftsbedingter Ungleichheiten im Bildungsund Erwerbssystem führen (vgl. Goldthorpe 1966). Aus modernisierungstheoretischer Sicht ist in Zusammenhang mit den Strukturen und Entwicklungen des Schweizer Bildungswesens anzunehmen, dass in der Schweiz vor allem in der Nachkriegszeit die in der Abfolge von Geburtskohorten gestiegene Teilhabe an weiterführender und höherer Bildung mit einer sukzessive abnehmenden sozialen Ungleichheit von Bildungschancen einhergegangen ist (Hypothese 1).

Aus konflikttheoretischer Sicht ist aber anzunehmen, dass dominante gesellschaftliche Statusgruppen ihre Privilegien auch im Zuge der Bildungsexpansion dadurch zu erhalten versuchen, dass sie den Bildungsaufsteigern aus unteren Sozialschichten den Zutritt zu sozial exklusiven Bildungsbereichen und folglich zu den prestigeträchtigen gesellschaftlichen Positionen verwehren (vgl. Bourdieu und Passeron 1971). Die soziale Schließung und damit die Aufrechterhaltung sozialer Ungleichheit von Bildungs- und Arbeitsmarktchancen gelingt ihnen unter anderem durch die Anhebung von Leistungsanforderungen für den Zugang zu höherer Bildung oder für den Erwerb von Bildungspatenten oder durch die Schaffung neuer exklusiver Ausbildungsmöglichkeiten und Bildungstitel (vgl. 
Collins 1979; Weber 1972, S. 577). Dazu gehören in der Schweiz die traditionelle Attraktivität des Berufsbildungssystems, die besonders für soziale Aufsteiger wenig riskant und aufwendig erscheint, und gleichzeitig die allgemein verbreitete Auffassung, dass die Gymnasialbildung und die Maturität (wie in Deutschland bis ca. 1970) ausschließlich zum aufwendigen und anspruchsvollen Studium an der Universität oder ETH führen soll. Aus Sicht der Konflikttheorie ist anzunehmen, dass trotz der Bildungsexpansion die soziale Ungleichheit von Bildungschancen im Allgemeinen und beim Erwerb der gymnasialen Maturität (und folglich beim Hochschulzugang) im Besonderen zumindest konstant geblieben ist (Hypothese 2).

Der „maximally maintained inequality thesis“ (MMI-These) zufolge verringern sich Bildungsdisparitäten dann nur noch durch die Sättigung der Bildungsnachfrage in den höheren Sozialschichten und das, womöglich mit zeitlicher Verzögerung, graduelle Aufholen sozial benachteiligter Gruppen (vgl. Raftery und Hout 1993). Obgleich unklar ist, wann eine Sättigung der Bildungsnachfrage einsetzt, bleibt diesem Ansatz zufolge durch das konstant bleibende schichttypische Bildungsverhalten die soziale Ungleichheit von Bildungschancen trotz mehr Bildungsgelegenheiten zunächst für eine historische Periode konstant und nimmt dann, weil dann nur noch die sozial benachteiligten Gruppen in höhere Bildung investieren, wieder ab. Der von Lucas (2001) vorgeschlagenen „effectively maintained inequality in education thesis“ (EMI-These) entsprechend, verschieben sich bei steigender Bildungsnachfrage aus den unteren und mittleren Sozialschichten sozial bedingte Bildungsungleichheiten in Richtung der Qualität bei der Auswahl von Bildungsinstitutionen, Bildungsgängen und Zertifikaten. So könnten im Zuge der Bildungsexpansion soziale Ungleichheiten von Bildungschancen beim Erwerb der Studienberechtigung auf bislang sozial exklusiven Schulen wie dem Gymnasium zunehmen, über die eine Studienberechtigung für die sozial noch exklusiveren Universitäten vergeben werden. Bei einer zunehmenden Inanspruchnahme des Gymnasiums durch soziale Bildungsaufsteiger ist diesem Ansatz zufolge nicht ausgeschlossen, dass die höheren Sozialschichten zum Ersten ausschließlich auf die gymnasiale Maturität setzen, zum Zweiten auf prestigeträchtige Hochschulen (wie etwa die Eidgenössischen Technischen Hochschulen (ETH) in der Schweiz oder im Ausland) ausweichen und zum Dritten sich verstärkt für ebenso prestigeträchtige und (immer noch) sozial exklusive Studienfächer wie etwa Medizin oder Jura (vgl. für die Schweiz: Becker 2012; für Deutschland: Becker et al. 2010) entscheiden. Als Folge davon würden sich Bildungsungleichheiten auf die Ebene der Hochschulen, und hierbei vor allem auf die sozial exklusiven Universitäten, verschieben und im Wesentlichen konstant bleiben. Ob diese von der MMI- und EMI-These prognostizierte Entwicklung von während der bedeutsamen Phasen der Bildungsexpansion konstant bleibender und dann abnehmender sozialer Ungleichheit von Bildungschancen auch für den Erwerb der gymnasialen Maturität zu beobachten ist, wird als Hypothese 3 empirisch zu überprüfen sein.

Zusätzlich zu diesen makrosoziologischen Erklärungsversuchen wurden in den letzten 15 Jahren strukturell-individualistische Theorien herangezogen, um über Mechanismen und Prozesse auf allen gesellschaftlichen Ebenen den Zusammenhang von Bildungsexpansion und Bildungsverhalten nach sozialer Herkunft und Geschlecht zu erklären (vgl. Breen und Goldthorpe 1997; Erikson und Jonsson 1996; Solga und Becker 2012). Diese Theorien bauen auf der von Boudon (1974) vorgeschlagenen Unterscheidung von 
primären und sekundären Effekten der sozialen Ungleichheit auf. Während sich der Zusammenhang von sozialer Herkunft, und übertragen auf Geschlechterdisparitäten im Bildungssystem, oder von Geschlecht und Schulleistungen (primärer Effekt) kaum in der Zeit gewandelt hat (vgl. Becker und Müller 2011; Erikson und Rudolphi 2010), dürfte eher der Wandel des schicht- oder geschlechtstypischen Bildungsverhaltens (sekundärer Effekt) den Zusammenhang von Bildungsexpansion und -ungleichheiten erklären (vgl. Becker 2003).

Wenn individuelle Bildungsentscheidungen auf mehr oder weniger rational kalkulierenden Abwägungen von subjektiv erwarteten Renditen und Kosten für Bildung basieren, dann sind vor allem Veränderungen der strukturellen und institutionellen Rahmenbedingungen, unter denen Akteure jeweils evaluieren, entscheiden und handeln, ausschlaggebend für Verlauf und Sozialstruktur der Bildungsexpansion (vgl. Breen et al. 2012). Deswegen ist von einer Kohortendifferenzierung der Bildungsexpansion auszugehen. Denn in Abhängigkeit von ihrer historischen Lagerung werden unterschiedliche Geburtsjahrgänge in jeweils unterschiedlicher Art und Weise durch vorherrschende gesellschaftliche Verhältnisse geprägt (vgl. Mayer und Huinink 1990). Somit müssten sich die Kohorten systematisch im Ausmaß und in der Sozialstruktur der Bildungsbeteiligung voneinander unterscheiden. Selbst wenn strukturelle und institutionelle Rahmenbedingungen in der historischen Periode variieren, dürften sie, aus der Perspektive des Lebensverlaufs betrachtet, hauptsächlich an den entscheidenden Bildungsübergängen im Lebenslauf, wie etwa dem Übergang in die Sekundarstufe I oder in die Sekundarstufe II, eine prägende Auswirkung auf den weiteren Bildungsverlauf haben, sodass für den Erwerb von Bildungszertifikaten (wie etwa der gymnasialen Maturität) Kohorten- über Periodeneffekte dominieren (vgl. Blossfeld 1988).

Des Weiteren sind steigende Bildungsbeteiligungen in allen Bevölkerungsschichten zu erwarten, wenn sich mehr Bildungsgelegenheiten ergeben (z. B. durch flächendeckenden Schul- und Hochschulbau, zunehmende Zahl von Lehrpersonen usw.). Hierbei dürfte entscheidend sein, ob diese aufgrund sinkender relativer Bildungskosten (z. B. zunehmender Wohlstand, Lehrmittelfreiheit usw.) oder steigender Renditen (z.B. Nachfrage der Arbeitgeber nach besser qualifizierten Arbeitskräften, höhere Löhne für besser qualifizierte Arbeitskräfte bei zunehmenden Qualifikationsanforderungen der beruflichen Tätigkeiten usw.) auch von einem zunehmenden Teil der Bevölkerung genutzt werden (können). Da auch in der Schweiz die nachobligatorische Bildung ein öffentliches Gut ist, von dessen Nutzung niemand ohne legitimen Grund ausgeschlossen werden kann (z. B. wegen unzureichender Schulleistungen), birgt eine zunehmende Bildungsbeteiligung der bislang in der höheren Bildung unterrepräsentierten Sozialschichten für sozial privilegierte Gruppen die Gefahr, dass höhere Bildungszertifikate ihre exponierte Funktion als Positionsgut einbüßen. Das ist dann der Fall, wenn die unteren Sozialschichten die Nachfrage nach höherer Bildung eher steigern als die sozial privilegierten Sozialschichten. In solchen Zeiten sinkt die soziale Ungleichheit von Bildungschancen zugunsten der benachteiligten Gruppen. Damit ihre, über sozial exklusive Bildung abgesicherten Statuspositionen nicht durch Bildungsaufsteiger gefährdet werden, sind dann die höheren Sozialschichten aufgrund der verstärkten Statuskonkurrenz gezwungen, wieder vermehrt in höhere Bildung ihrer Kinder und Kindeskinder zu investieren (vgl. Boudon 1974; Lamprecht und Stamm 1966; Meylan 1966, S. 11). In solchen Phasen dürfte sich 
die soziale Ungleichheit von Bildungschancen wieder deutlich verstärken. Da sich aber die Bildungsentscheidungen in Abhängigkeit der Klassenlage nicht grundlegend ändern (vgl. Breen und Goldthorpe 1997), führt eine Bildungsexpansion nicht zwangsläufig zum Abbau von Bildungsungleichheiten, sondern es kann Zyklen von abnehmenden und wieder ansteigenden Bildungsungleichheiten geben, je nachdem, ob in der Abfolge von Generationen der Wettbewerb um Bildung als positionales Mittel für den Statuserhalt abnimmt oder zunimmt. Kurzum: Im Unterschied zur Modernisierungstheorie werden im Zuge der Bildungsexpansion keine linear abnehmenden Bildungsungleichheiten erwartet, sondern, infolge sich wandelnder Rahmenbedingungen, azyklische Abfolgen von mehr oder weniger großen Bildungsungleichheiten als unvorhergesehene Folge des absichtsvollen, zwischen den Sozialschichten differierenden Bildungsverhaltens.

Dem strukturell-individualistischen Ansatz zufolge müssten bei einer informellen, nicht kodifizierten Kontingentierung der Gymnasialplätze, wie dies in der Schweiz in den letzten Jahrzehnten kontinuierlich der Fall war, die Jugendlichen aus den höheren Sozialschichten und bildungsnäheren Familien wegen des Zusammenspiels von primären und sekundären Herkunftseffekten generell im Vorteil sein, sodass herkunftsbedingte Bildungsungleichheiten zumindest konstant bleiben. Bei einer von privilegierten Statusgruppen befürchteten Gefährdung der gymnasialen Maturität und des daran gebundenen exklusiven Zugangs zu den Universitäten als Positionsgut infolge einer Erhöhung der Gymnasialquote sollte sich die soziale Ungleichheit beim Erwerb der gymnasialen Maturität gar verschärfen (Hypothese 4).

Unabhängig von ihrer sozialen Herkunft könnten die Frauen aber im Zuge verbesserter Bildungsgelegenheiten angesichts ihrer ohnehin besseren Schulleistungen eben durch die höheren Zulassungsquoten zum Gymnasium auf der Sekundarstufe II ihre Rückstände bei der höheren Bildung gegenüber den Männern deutlich ausgleichen (Hypothese 5). Die Anreize für dieses Bildungsverhalten ergeben sich aus der im Zuge der Tertiarisierung der Berufsstruktur in den letzten Jahrzehnten gestiegenen Nachfrage nach qualifizierten und hoch qualifizierten Arbeitskräften für berufliche Tätigkeiten im Dienstleistungssektor, die typischerweise von Frauen ausgeübt werden und für die in den letzten Jahrzehnten die Qualifikationsanforderungen angehoben worden sind. Ein für die Schweiz typisches Beispiel ist die im Laufe der letzten 10 bis 20 Jahre eingeführte Regel, dass für die Ausbildung in vielen Dienstleistungsberufen wie etwa in dem der Primarschullehrerin die gymnasiale Maturität erforderlich ist (SKBF 2011, S. 122).

\section{Datenbasis, Variablen und statistisches Verfahren}

\subsection{Datenbasis}

Die empirischen Analysen basieren auf harmonisierten Daten der Schweizer Volkszählungen in den Jahren 1970, 1980, 1990 und 2000 (vgl. Sheldon 2005). Seit 1850 wird jeweils im Abstand von 10 Jahren ein Zensus durchgeführt (vgl. Busset 1993). Diese in regelmäßigen Abständen durchgeführten Vollerhebungen erlauben wegen großer Fallzahlen differenzierte Analysen der Bildungsexpansion unter besonderer Berücksichtigung demografischer, ökonomischer und sozialstruktureller Prozesse. Die Gesamtheit der 
Volkszählungen bilden Personen, Haushalte und Erwerbspersonen der am wirtschaftlichen und zivilrechtlichen Wohnsitz bemessenen Wohnbevölkerung. Im Erhebungsprogramm sind neben Haushaltsmerkmalen und abgeleiteten Variablen (wie etwa sozioprofessionelle Kategorien oder Referenzperson des Haushaltes) für unsere Fragestellung relevante Individualmerkmale wie etwa Geburtsdatum und -land, Geschlecht, Ausbildung, Beruf oder berufliche Stellung und Stellung im Haushalt verfügbar. Somit ist es möglich, die Bildungsexpansion in der Schweiz für das 20. Jahrhundert im Allgemeinen und für die Zeit nach $1950 \mathrm{im}$ Besonderen theoriegeleitet nachzuzeichnen. Stichtag der Volkszählung (VZ) ist in der Regel Anfang Dezember (z. B. 5. Dezember 2000 bei der VZ 2000). ${ }^{8}$

Bei den bivariaten Analysen, der Beschreibung der Bildungsexpansion und der Entwicklung von Bildungsabschlüssen im Zeitverlauf, beschränken wir uns für jede der VZ auf Frauen und Männer im Alter von 20 bis 89 Jahren. Die über 90-jährigen Personen stellen eine kleine Minderheit dar, die mit einer Fallzahl deutlich unter 100000 Personen quantitativ kaum ins Gewicht fällt. Zudem weisen sie einen relativ hohen Anteil fehlender Angaben für den höchsten Bildungsabschluss von mehr als 25\% auf. Bei den unter 20-Jährigen ist der Bildungsverlauf noch nicht vollständig abgeschlossen, sodass bei dieser Gruppe die Dynamik der Bildungsexpansion unterschätzt wird. Insgesamt stehen für das Jahr 1970 Informationen für rund 4,3 Millionen Personen zur Verfügung, für 1980 für rund 4,6 Millionen, für 1990 rund 5,2 Millionen und schließlich für 2000 rund 5,5 Millionen Personen.

Für die Überprüfung der These dauerhafter Bildungsungleichheit mittels multivariater Analysen hingegen werden Frauen und Männer im Alter von 18 bis 21 Jahren herangezogen, die noch im Elternhaus leben. Da in der VZ die soziale Herkunft nicht direkt für alle Personen erfasst wird, kann sie nur über die Haushaltsstruktur für die noch im Elternhaus lebenden Kinder rekonstruiert werden. Weil die abhängige Variable der Erwerb der gymnasialen Maturität versus andere Schulabschlüsse ist, wurde dieses Altersintervall verwendet. Mit 18 Jahren kann in der Regel frühestens die allgemeine Studienberechtigung (gymnasiale Maturität) erworben werden, und für höhere Lebensalter werden spätere Einschulung, Klassenwiederholungen, Schul- und Schulformwechsel usw. berücksichtigt. Aufgrund noch geringer Fallzahlen für die im Jahre 1993 eingeführte Berufsmaturität (vergleichbar mit der Fachhochschulreife und dem Fachabitur in Deutschland) und ihrer fehlenden Ausweisung kann sie bei den Analysen für die VZ 2000 noch nicht berücksichtigt werden. Die Teilgesamtheit der 18- bis 21-jährigen Personen ist einer Überprüfung zufolge zwar selektiv in Bezug auf den Bildungsabschluss und das Geschlecht, wonach durchaus zu Recht angenommen werden kann, dass Personen mit einer Berufsmaturität größtenteils bereits aus dem Elternhaus ausgezogen sind. Weil für sie deswegen Informationen zu ihrer sozialen Herkunft fehlen, können sie nicht weiter berücksichtigt werden. Entscheidend für unsere Analysen ist jedoch, dass für beide Geschlechter bei allen

8 Bis zur VZ 2000 wurden die Informationen mittels standardisierter Fragebogen erhoben. Seit 2010 wird die VZ jährlich registerbasiert (Einwohnerregister und andere prozessproduzierte Verwaltungsdaten mit 31. Dezember 2010 als Stichtag) mit ergänzenden Stichprobenerhebungen durch das Bundesamt für Statistik durchgeführt. Lediglich 5\% der Bevölkerung werden schriftlich oder telefonisch befragt. Diese Daten stehen jedoch noch nicht der „scientific community“" zur Verfügung 
Messzeitpunkten ein sehr schwacher Zusammenhang zwischen Bildungsabschluss und Haushaltsstatus besteht. Demnach liegt in Bezug auf Bildung, Geschlecht und Haushaltsform keine selektive Stichprobe vor, wenn lediglich diejenigen Personen berücksichtigt werden können, die noch im Elternhaus sind. Somit dürften die Ergebnisse zum Erwerb der gymnasialen Maturität hinreichend reliabel sein. ${ }^{9}$

\subsection{Variablen}

Eine zentrale Variable ist der erworbene Bildungsabschluss. Für die Analysen werden die ursprünglichen Bildungsabschlüsse in den harmonisierten Datensätzen verwendet. Folgende Kategorien werden unterschieden: 1) keine abgeschlossene Schul- und Berufsbildung, 2) maximal obligatorische oder berufsvorbereitende Schule (einschließlich nicht abgeschlossener Besuch der Mittel- oder Diplommittelschule), 3) Berufslehre oder Vollzeit-Berufsschule, 4) Maturität, 5) höhere Berufsausbildung und höhere Fachschule, 6) Hochschule (Universität, Fachhochschule und andere Hochschulen wie ETH oder Pädagogische Hochschule). Eine zum Messzeitpunkt noch nicht abgeschlossene Ausbildung oder fehlende Angabe zum Bildungsniveau wird zwecks Vermeidung von Stichprobenverzerrung durch selektiv fehlende Antworten ebenfalls als gültiger Wert berücksichtigt.

Bei den multivariaten Analysen werden als abhängige Variable für die 18- bis 21-Jährigen die Chancen untersucht, mit der (gymnasialen) Maturität eine Studienberechtigung zu erwerben. Die Referenzkategorie sind niedrigere Schul- und Ausbildungsabschlüsse, Abschluss einer höheren Berufsausbildung oder keine Angabe zum erworbenen Bildungsabschluss. Offensichtliche Fehlzuordnungen bei der Harmonisierung der Zensusdaten durch das Bundesamt für Statistik werden von der Analyse ausgeschlossen (vgl. Sheldon 2005). Bei multivariaten Analysen wird das höchste Bildungsniveau eines Elternteils als Indikator für soziale Herkunft verwendet.

Ein weiterer Indikator für die soziale Herkunft ist die Klassenlage des Elternhauses. Sie wird mit dem bewährten Klassenschema von Erikson et al. (1979) abgebildet. Dem Vorschlag von Lepsius (1979) folgend, wird mit der Versorgungsklasse eine soziale Klasse der Nichterwerbstätigen und Rentner berücksichtigt, die ihren Lebensunterhalt durch Partnerschaft oder sozialstaatliche oder -politische Transfereinkommen bestreiten. Bei der Versorgungsklasse werden Rentnerinnen und Rentner sowie Erwerbslose oder sich noch in Ausbildung befindliche oder im Haushalt tätige Personen unterschieden.

Der Migrationsstatus wird zunächst anhand des Geburtsortes bemessen und dient als Kontrollvariable. Unterschieden wird die Geburt im Ausland oder, bei multivariaten Analysen als Referenzkategorie, in der Schweiz. Zudem wird unter Berücksichtigung der Einwanderungsgeschichte der Schweiz danach unterschieden, in welchem Land die Personen geboren wurden (vgl. Beck et al. 2010).

9 Die Zusammenhangsmaße Cramér's V liegen für alle VZ bei 0,34 und es ist nicht möglich, anhand der Kenntnis des Bildungsniveaus mit ausreichender Sicherheit vorherzusagen, ob die Personen bereits aus dem Elternhaus ausgezogen sind. Die entsprechenden Tabellen können auf Anfrage bei den Autoren bezogen werden. 


\subsection{Statistisches Verfahren}

Für die Schätzung der Bildungschancen der 18- bis 21-jährigen Personen wird die logistische Regression angewandt (vgl. Long 1997). Als Einflussgrößen für die Bildungschancen werden die „average marginal effects“ (AME) berechnet. Die AME erlauben den Vergleich der Koeffizienten zwischen Gruppen sowie über die Zeit hinweg (vgl. Best und Wolf 2012; Hinz und Auspurg 2011).

Da wir mit Grundgesamtheiten statt mit Zufallsstichproben operieren, werden keine Signifikanztests vorgenommen, weil jeder beobachtete Unterschied real und nicht zufällig ist. Zumindest wird das Signifikanzniveau, das wir mit einem Prozent als Minimum festgelegt haben, nicht inhaltlich interpretiert. Allenfalls sind sie eine „objektive“ Hilfe bei der subjektiven Beurteilung, ob es sich jeweils um sehr bedeutsame Unterschiede handelt.

\section{Empirische Ergebnisse}

\subsection{Bildungsexpansion in der Schweiz des 20. Jahrhunderts}

Wird die Entwicklung des Bildungsstandes der Bevölkerung im Querschnitt für die einzelnen Schweizer VZ betrachtet, so lässt sich die Bildungsexpansion zunächst anhand der sukzessiven Höherqualifikation in der historischen Periode rekonstruieren (Abb. 1). Von Zensus zu Zensus nimmt der Anteil der zwischen 20- und 89-jährigen Bürgerinnen und Bürger von $45 \%$ im Jahre 1970 über $41 \%$ (1980) und $31 \%$ (1990) auf $25 \%$ im Jahre $2000 \mathrm{ab}$, die maximal über eine obligatorische Schulbildung verfügen und allenfalls eine

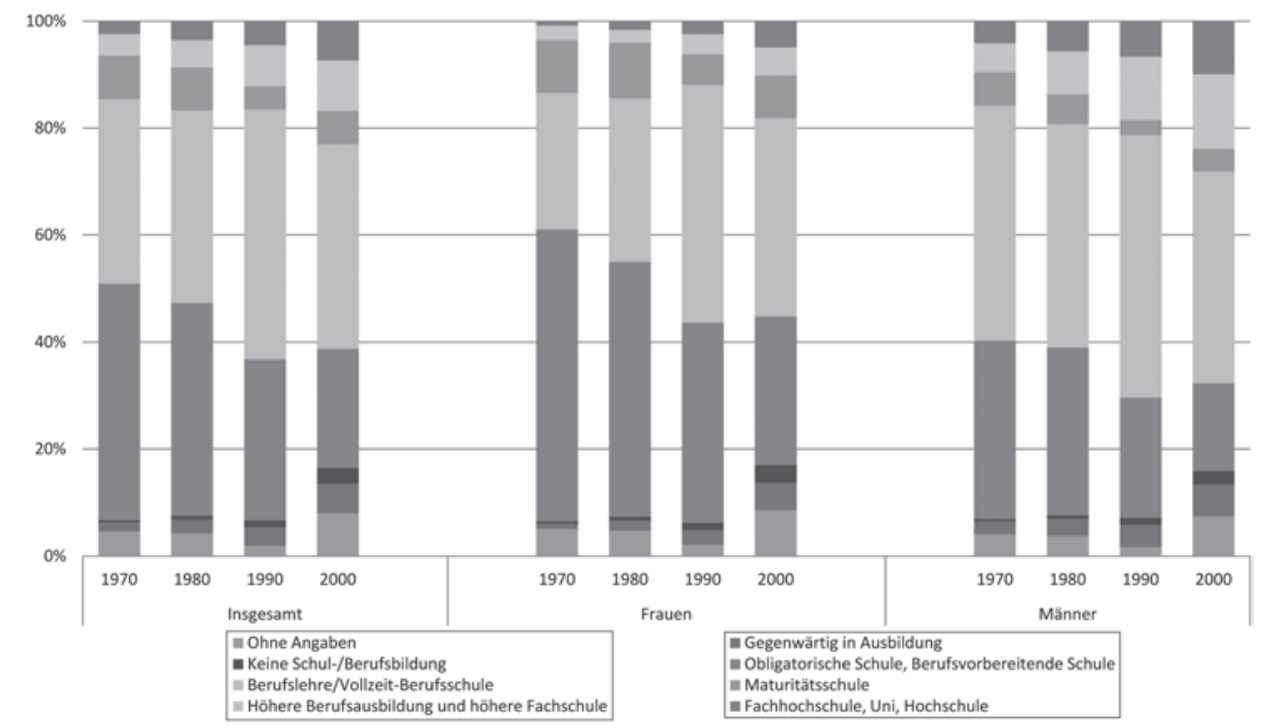

Abb. 1: Entwicklung des Bildungsstandes in der Schweiz - nur 20- bis 89-jährige Personen 
berufsvorbereitende Schule besucht haben. Hingegen steigt die Zahl von Personen, die eine sekundäre Berufsausbildung abgeschlossen haben (1970: 35\%; 1980: 36\%; 1990: 47\%; 2000: 38\%), und auch der Anteil in der Bevölkerung, der eine höhere Berufsausbildung (von $4 \%$ im Jahre 1970 auf $10 \%$ im Jahre 2000) oder ein Hochschulstudium (von $5 \%$ auf rund $10 \%$ ) absolviert hat. Zählt man die berufliche Lehre und höhere Berufsausbildung zusammen, so weist bei den letzten beiden Zensen mindestens die Hälfte der Bevölkerung im Alter zwischen 20 und 89 Jahren eine abgeschlossene Berufsausbildung vor. Diese Bildungsabschlüsse, die eine entsprechende Schulbildung voraussetzen, sind in der Nachkriegszeit zum Standard geworden. Hingegen ist für die gesamte Bevölkerung betrachtet der Hochschulabschluss eher die große Ausnahme (Anstieg von 2 auf 7\% in der gesamten Periode).

Der Prozess der Höherqualifikation ging auch, wie aus Sicht der strukturell-individualistischen Theorie vermutet (Hypothese 5), mit der Verringerung der Bildungsrückstände von Frauen gegenüber den Männern einher. Vor allem bei der sekundären und tertiären Berufsausbildung haben die Frauen in den letzten Jahrzehnten aufgeholt. Auffallend ist für den letzten Messzeitpunkt die weiterhin im internationalen Vergleich unterdurchschnittliche Akademikerquote, deren Anhebung einer der Gründe für die bildungspolitische Initiierung der Bildungsexpansion in der Schweiz war. ${ }^{10}$ Trotz der Verdopplung der Zahl von Hoch- und Fachhochschulabsolventen in den letzten Jahren liegt die Akademikerquote in der Schweiz weiterhin unter dem internationalen OECD-Durchschnitt.

Die zwischen 1990 und 2000 gesunkenen Anteile für sekundäre Berufsausbildung und Maturität weisen auf methodische Probleme dieser Querschnittbetrachtung hin. So wird durch die Vermischung von Altersgruppen, die sich in jeweils unterschiedlichen Stadien des Bildungsverlaufs befinden, sowohl die Dynamik der Bildungsexpansion als auch die Kohortendifferenzierung dieses Prozesses unterschätzt. ${ }^{11}$ Die Vermischung der Alters- und Lebenszykluseffekte, Kohorteneffekte und Periodeneffekte durch die komparativ-statische Betrachtungsweise führt in der Regel zu irreführenden Interpretationen der Struktur und Dynamik der Bildungsexpansion. Deswegen wird als ein Ausweg die Bildungsexpansion in der Schweiz differenziert für die Altersgruppe der 30- bis 39-jährigen Personen betrachtet (Abb.2). Diese Altersgrenzen werden gewählt, um sicherzugehen, dass die Schul- und Berufsausbildung und dann wahrscheinlich auch die höhere Berufsausbildung sowie das Hochschulstudium bereits abgeschlossen wurden und auch zusätzliche Ausbildungen berücksichtigt werden.

Für diese Alterskohorten sehen wir einen sukzessiven Anstieg für das höhere Bildungsniveau ab der Maturität von 17\% im Jahre 1970 über 22 bzw. $23 \%$ in den Jahren 1980

$10 \mathrm{Ob}$ der zunehmende Anteil an fehlenden Angaben zum Bildungsabschluss eine der Folgen der Bildungsexpansion ist, indem die besser gebildeten Befragten die Möglichkeiten und Risiken für die Verweigerung von Angaben eher abschätzen können, kann mit den verfügbaren Daten nicht abgeschätzt werden.

11 Des Weiteren werden Auswirkungen demografischer Prozesse, insbesondere der kohorten- und bildungsdifferenziellen Fertilität sowie der differenziellen Mortalität nach Geburtsjahr, Bildung und Geschlecht auf die Bildungsexpansion unterschlagen. Schließlich werden im Querschnitt die Effekte der sozial selektiven Immigration und Auswanderung auf den Bildungsstand der Bevölkerung nicht erfasst. Mangels prospektiver Längsschnittdaten für längere historische Zeiträume können wir diese Problematik nicht weiter berücksichtigen. 


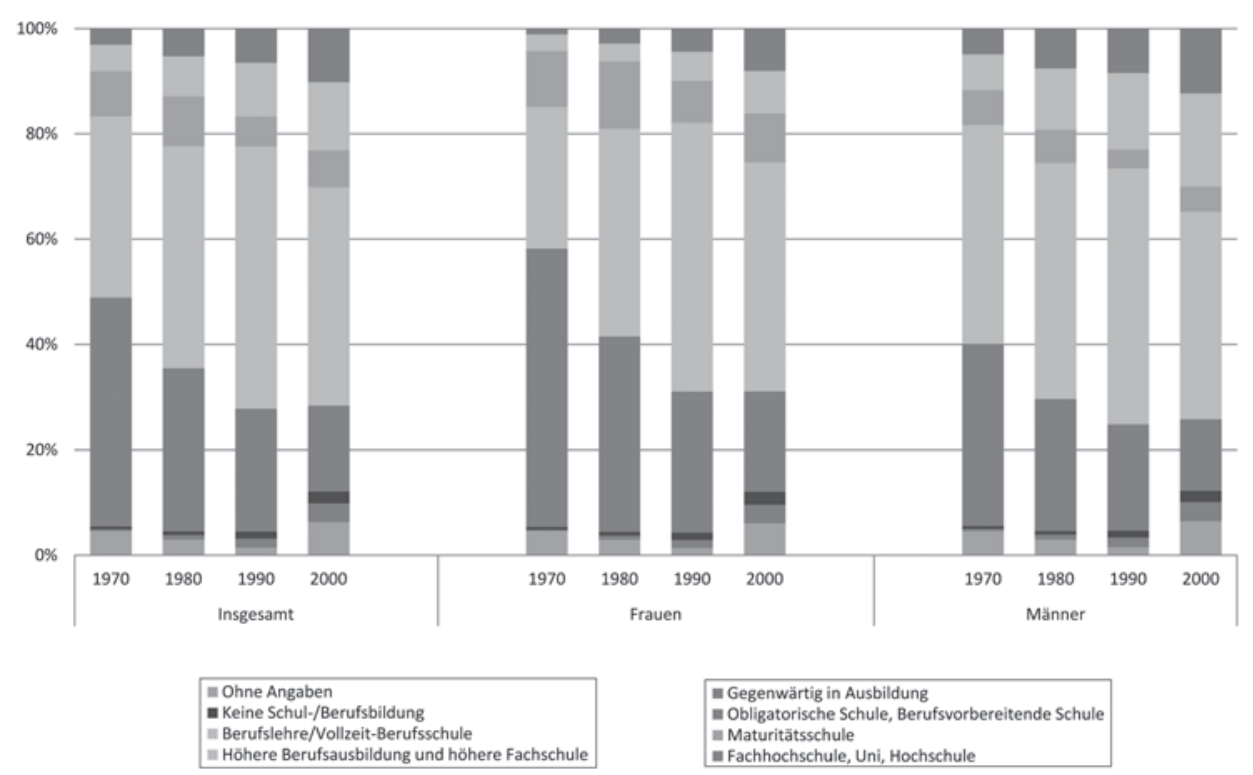

Abb. 2: Entwicklung des Bildungsstandes in der Schweiz - nur Personen im Alter von 30 bis 39 Jahren

und 1990 auf über 30\% im Jahre 2000. Hier ist zu berücksichtigen, dass der Ausbau des gymnasialen Bildungswesens in den 1960er Jahren einsetzte, sodass die Folgen dieser Ausdehnung erst für die jüngeren Jahrgänge ersichtlich sein dürften, wenn man deren Bildungsverläufe zeitlich kontinuierlich verfolgen könnte, wie dies mit ereignisorientierten, prospektiv erhobenen Verlaufsdaten möglich ist. Aber erste Anzeichen dafür sind schon für die Jahrgänge der zwischen 1961 und 1970 Geborenen im Jahre 2000 erkennbar. Es ist ersichtlich, dass die gymnasiale Maturität für die meisten Personen eben lediglich eine Zwischenstation im Bildungsverlauf auf dem Wege zur höheren Berufsausbildung und zum Hochschulstudium ist. Deswegen ist in der tertiären Ausbildung, wie aus strukturell-individualistischer Sicht prognostiziert, eine kohortendifferenzierende Höherqualifikation zu verzeichnen, da die Maturandinnen und Maturanden ihren Bildungsverlauf mit dem Hochschulstudium fortsetzen. Das entspricht auch dem Verständnis von Maturität in der Schweiz. Im Unterschied zum Abitur in Deutschland ist sie kein omnipotentes Zertifikat, das alle Bildungsoptionen offenhält, sondern das Eintrittsticket für die Hochschulbildung. Der Anteil von Personen im Alter von 30 bis 39 Jahren mit maximal obligatorischer Bildung sinkt in diesem Zeitraum deutlich von 44 auf 18,5\%, während derjenige mit einer sekundären Berufsausbildung ebenso deutlich bis zum Jahre 1990 von 34 auf 50 \& zunimmt. Danach sinkt deren Anteil auf $41 \%$ im Jahre 2000, und es ist zu vermuten, dass dieser Trend über die nachfolgenden Jahre anhalten wird.

Sind diese Entwicklungen in der Struktur und Dynamik der Bildungsexpansion auch für die altersgleichen Frauen und Männer gleichermaßen zu beobachten? In der Nachkriegszeit haben sich die anfänglichen Rückstände der Frauen im Alter von 30 bis 39 Jahren gegenüber den Männern im gleichen Alter sukzessive verringert (Abb. 2). Bei den mittleren und höheren Bildungsabschlüssen verschwinden die Geschlechterunterschiede 
gänzlich. Erst für das Jahr 2000 deutet es sich an, dass beim Erwerb eines Hochschulabschlusses die Frauen zu den Männern aufgeschlossen haben. Damit wird der strukturellindividualistische Erklärungsansatz (Hypothese 5) weiter unterstützt.

Unsere bivariaten Analysen bestätigen frühere Studien, wonach in der Schweiz seit Ende des 19. Jahrhunderts und noch deutlicher seit 1950 eine Bildungsexpansion stattgefunden hat. Im Vergleich zu anderen Ländern ist ihre Dynamik tatsächlich als „,träge“ zu bezeichnen. Ihre Struktur entspricht in keiner Weise den modernisierungstheoretischen Vorstellungen einer „Bildungsrevolution“, sondern eher der einer graduellen, kohortendifferenzierenden Höherqualifikation infolge sukzessiver Umschichtungen von einer in die nächsthöhere Bildungsstufe. Dieser Befund spricht eher für die theoretischen Überlegungen nach Boudon (1974). In den jüngeren Geburtskohorten konnten zudem die Frauen ihre Rückstände im Bildungssystem gegenüber den Männern mehr als nur ausgleichen. Welche Folgen alle diese Entwicklungen für Ungleichheiten beim Erwerb der Studienberechtigung haben und hatten, soll in einem weiteren Schritt untersucht werden.

\subsection{Entwicklung der sozialen Ungleichheit von Bildungschancen in der Schweiz}

Mittels binärer logistischer Regression wird für die 18- bis 21-jährigen Frauen und Männer zu den Zeitpunkten der VZ 1970, 1980, 1990 und 2000 untersucht, inwiefern Geschlecht, soziale Herkunft und Migrationshintergrund mit der Chance einhergehen, die gymnasiale Maturität zu erwerben. ${ }^{12}$ Im Vordergrund steht hierbei die eine Frage, ob sich herkunftsbedingte und geschlechtstypische Bildungsungleichheiten im Zuge der Bildungsexpansion generell verringert haben. Die andere Frage ist, ob strukturelle und institutionelle Veränderungen des Schweizer Bildungssystems in den 1990er Jahren gar zur verschärften Ungleichheit beim Erwerb der gymnasialen Maturität beigetragen haben.

Bei Kontrolle des Geschlechts und des Migrationshintergrunds ist eine deutliche Abnahme der Ungleichheiten nach sozialer Herkunft bis 1990 und dann wieder eine Zunahme der herkunftsbedingten Bildungsungleichheiten im Jahre 2000 festzustellen (Tab. 1). ${ }^{13}$ Dieses Muster widerspricht eindeutig der Modernisierungstheorie (Hypothese 1), im Großen und Ganzen auch dem konflikttheoretischen Ansatz (Hypothese 2) sowie der MMI- oder EMI-These (Hypothese 3), bestätigt aber die Annahmen der strukturell-individualistischen Theorie (Hypothese 4). Trotz Bildungsexpansion sind bei zurückge-

12 Die Volkzählungsdaten erlauben für das Jahr 2000 keine Unterscheidung nach unterschiedlichen Maturitätstypen. Dadurch kann die Zunahme der sozialen Ungleichheit beim Erwerb der gymnasialen Maturität nicht durch einen selektiven Zustrom in die damals noch neue Berufsmaturität erklärt werden. Allerdings ist bei Kenntnis einer bereits abgeschlossenen beruflichen Ausbildung der potenzielle Anteil von Personen, die eine Berufsmaturität erworben haben könnten, wegen vergleichsweise früherem Auszug aus dem Elternhaus gering, sodass auch für das Jahr 2000 hauptsächlich die gymnasiale Maturität gemeint ist, wenn der Abschluss mit Maturität bezeichnet wird.

13 Worauf die zunehmende Ungleichheit der Migranten aus Portugal, Türkei und Ex-Jugoslawien in der zweiten Generation basiert, kann mit den Daten nicht überprüft werden. Vermutlich sind es die Kinder von Kriegsflüchtlingen, die vor dem Zerfall Jugoslawiens (Slowenienkrieg, Kroatienkrieg und mehrjähriger Bosnienkrieg) geflohen sind, die besonders benachteiligt sind im Schweizer Bildungssystem. 


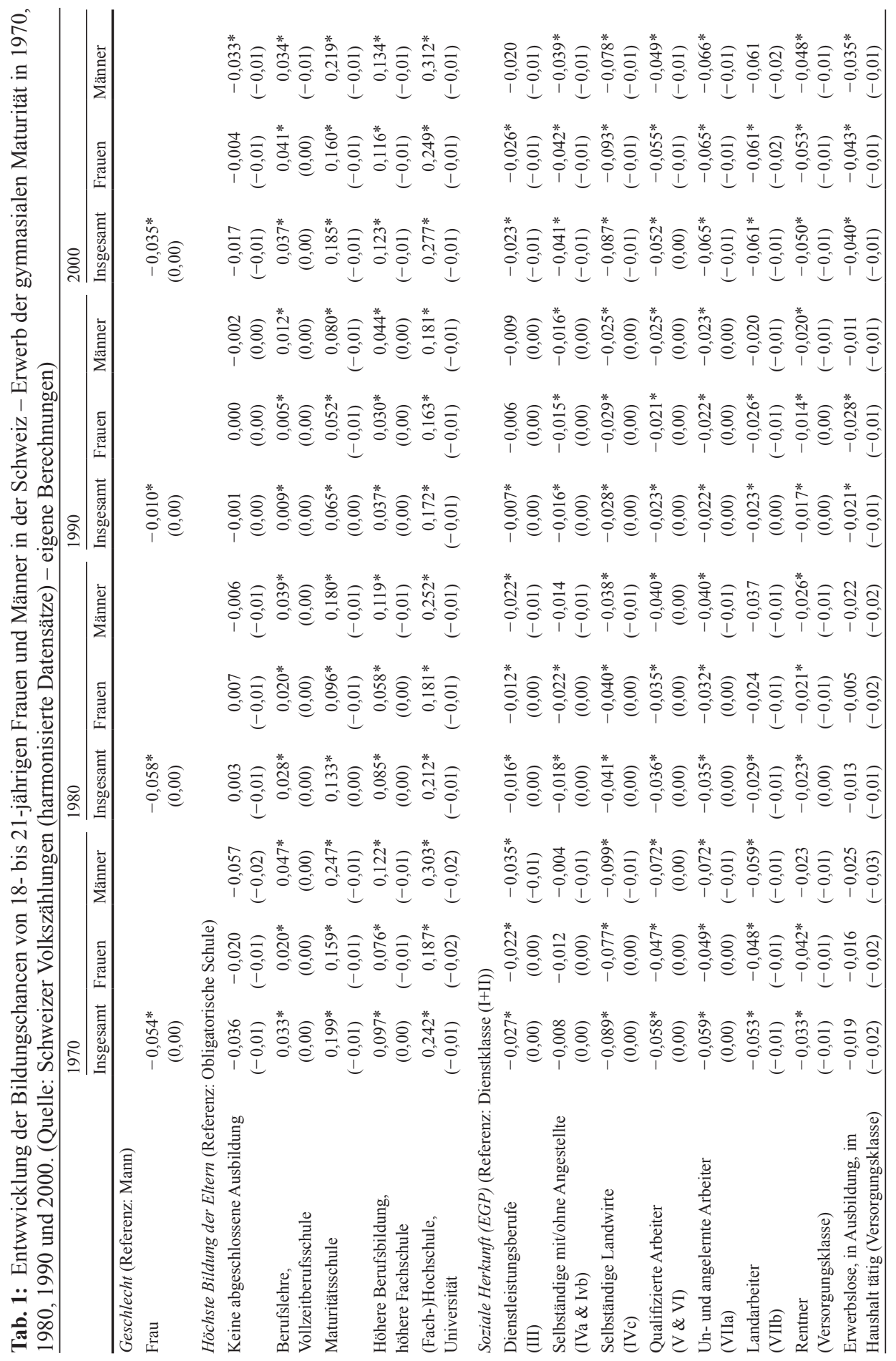




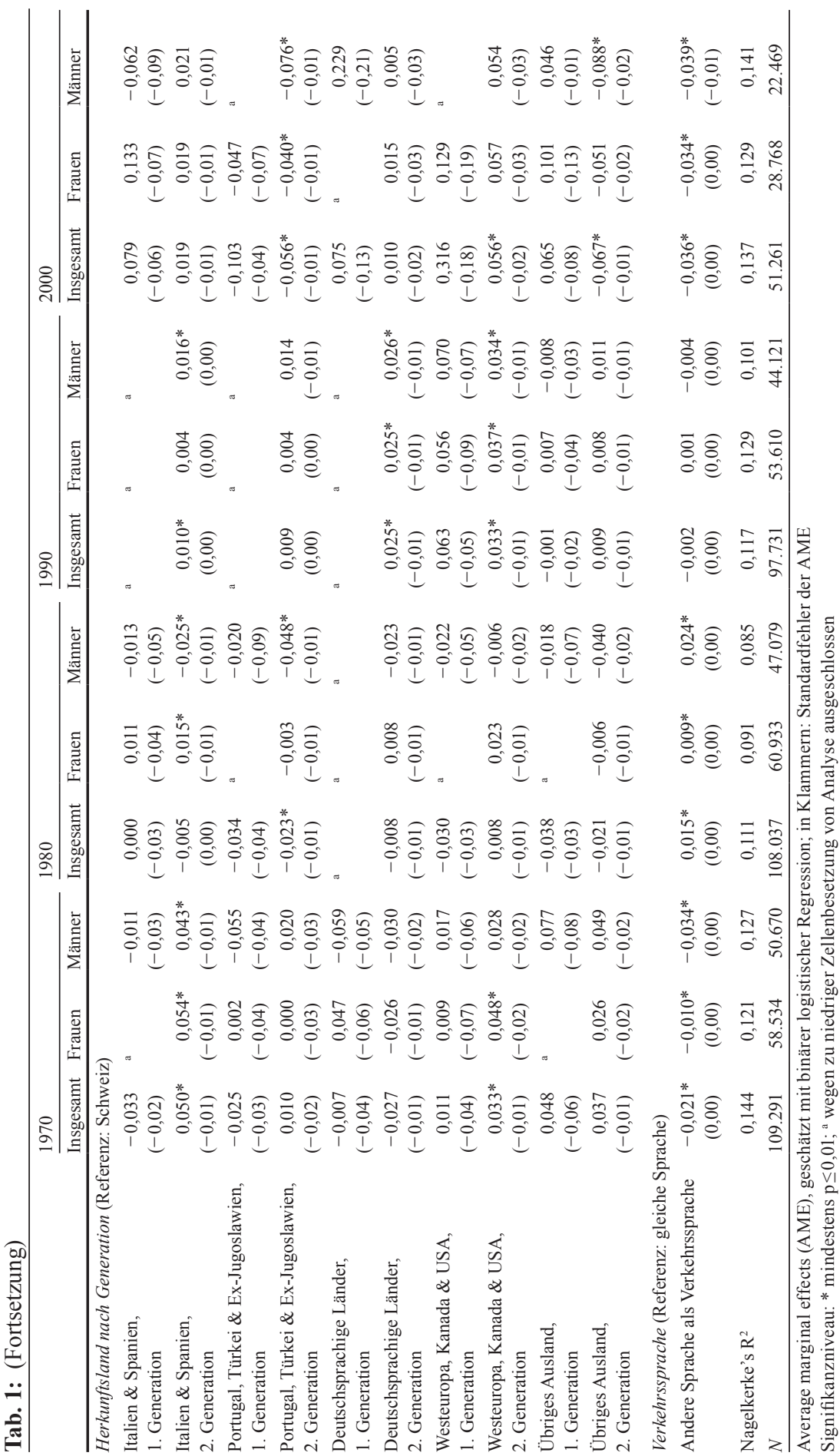


henden Bildungsungleichheiten in den 1970er und 1980er Jahren die Disparitäten nach sozialer Herkunft für die 18- bis 21-jährigen Frauen und Männer in der jüngeren Gegenwart immer noch deutlich. Sowohl die Ungleichheiten nach dem Bildungsniveau der Eltern als auch die Ungleichheiten nach der Klassenlage des Elternhauses nahmen bis 1990 ab. Davon profitierten bei Frauen wie bei Männern bislang sozial benachteiligte Gruppen. Allerdings gab es dann weiterhin eine ausgeprägte und bis zum Jahre 2000 wieder zunehmende intergenerationale Reproduktion bei der Maturität: Kinder von Eltern mit (mindestens) Maturitätsniveau erwarben selbst wiederum eher diesen Schulabschluss als Kinder niedrig gebildeter Eltern. ${ }^{14}$ Zudem sind die Abstände zwischen den Elternhäusern mit und den Elternhäusern ohne Hochschulabschluss, und für diesen Abschluss ist die Maturität institutionelle Voraussetzung, konstant markant. Diese Befunde gehen durchaus konform mit der konflikttheoretischen (Hypothese 2) und der strukturell-individualistischen Erklärung (Hypothese 4). Diese Hypothesen werden dadurch zusätzlich bekräftigt, dass die soziale Schließung der gymnasialen Maturität zusätzlich zur Kontingentierung der Maturitätsquote vor allem mit der 1993 eingeführten Berufsmaturität und der Umstellung auf ein binäres Hochschulsystem (Einführung von Fachhochschulen im Jahre 1995) verbunden ist. Zumindest kann hier nicht ausgeschlossen werden, dass die wieder angestiegene soziale Exklusivität beim Erwerb der gymnasialen Maturität mit einer gestiegenen Ablenkungswirkung für die unteren und mittleren Sozialschichten in den 1990er Jahren zusammenhängt. Das wiederum würde eindeutig für die strukturellindividualistische Erklärung sprechen.

Betrachten wir abschließend die für Frauen und Männer separat vorgenommenen Analysen. Ebenfalls deutlich zurückgegangen sind, bei Kontrolle der sozialen Herkunft und Migration, bis zum Jahre 1990 die Geschlechterdisparitäten beim Erwerb der Maturität, die dann aber wieder zulasten der Frauen zunahmen. Dieser Befund ist unerwartet und widerspricht der Hypothese 5, bei der von einer kontinuierlichen Abnahme der geschlechtstypischen Bildungsungleichheiten, auch beim Erwerb der gymnasialen Maturität, ausgegangen wurde.

Die Entwicklung nach sozialer Herkunft verläuft bei beiden Geschlechtern identisch. Herkunftseffekte nehmen gleichermaßen für Frauen und Männer ab. Einerseits waren, vom ersten Messzeitpunkt im Jahre 1970 aus betrachtet, bei den Frauen die Effekte der sozialen Herkunft schon immer geringer als bei den Männern. Andererseits bleiben bei der Angleichung von Herkunftseffekten (nach elterlichem Bildungsstand und Klassenlage des Elternhauses) die Geschlechterunterschiede bestehen. Worauf diese Entwicklung in der Schweiz beruht, ob sie sowohl auf geschlechtstypische Unterschiede bei den

14 Allerdings könnte diese Entwicklung der Ab- und Zunahme von Bildungsungleichheiten ein methodisches Artefakt sein. Es käme zustande, wenn ein wachsender Anteil der hier betrachteten Altersgruppe immer länger in der Ausbildung verbleibt und dadurch der Erwerb der Maturität zum Messzeitpunkt sozial selektiv unterschätzt wird. Dass dies möglich sein könnte, lässt sich am deutlichen Rückgang der Fallzahlen bei der Volkszählung 2000 ablesen, der nicht demografisch bedingt ist. Zusätzliche Analysen unsererseits haben zwar eine immer längere Ausbildungszeit in der Kohortenabfolge und damit eine zunehmende Zahl von „missing values“ für die abhängige Variable belegt. Die Berücksichtigung dieser fehlenden Fälle in der abhängigen Variable hat für die gesamte Entwicklung die gleichen Ergebnisse zutage gebracht wie die hier berichtete „complete case analysis“. 
schulischen Leistungen als auch auf jeweils unterschiedliche Bildungsentscheidungen zurückzuführen sind, kann mit diesen Daten ebenso wenig wie mit fast allen Datensätzen, die in der Schweiz der „scientific community“ zugänglich sind, nicht beurteilt werden. Vermutlich spielen verschiedene Entwicklungsstränge im Elternhaus, im Bildungssystem, auf dem Arbeitsmarkt, in der Partnerschaft, in der Planung von Familiengründung, beim Wohlstand, bei den Dienstleistungsangeboten und sonstigen Lebensplänen gewichtige Rollen. Deren komplexes Zusammenspiel müsste anhand von informationsreichen Lebensverlaufsdaten unterschiedlicher Geburtsjahrgänge untersucht werden.

\section{Zusammenfassung und Schlussfolgerung}

Ziel des vorliegenden Beitrages ist es, für die Schweiz im 20. Jahrhundert den Zusammenhang von Bildungsexpansion und sozialer Ungleichheit von Bildungschancen nach sozialer Herkunft und, als Spezialfall für diesen Zusammenhang, nach dem Migrationsstatus sowie nach Geschlecht empirisch differenziert zu beschreiben. Für die empirische Analyse wurden Daten der Volkszählungen in den Jahren 1970, 1980, 1990 und 2000 herangezogen. Im Vordergrund stand hierbei die Frage, ob die von Blossfeld und Shavit (1993) auch für die Schweiz vertretene These dauerhafter Bildungsungleichheit weiterhin aufrechterhalten werden kann.

Folgende Antworten können auf die Fragen, die die empirischen Analysen geleitet haben, geliefert werden. Wie veränderte sich in der historischen Periode, über die Abfolge von Geburtskohorten und über deren Lebensverläufe betrachtet die Verteilung von erworbenen Bildungsabschlüssen? Über die historischen Perioden im 20. Jahrhundert hinweg erfolgte in der Tat eine im Vergleich zu europäischen Ländern wie Schweden oder den Niederlanden zögerlich verlaufende Bildungsexpansion. Die graduelle Höherqualifikation mit Umschichtungen von niedriger in die mittlere und dann in die höhere Bildung ging über die Abfolge von Kohorten vonstatten. Und wer profitierte hierbei besonders von der Bildungsexpansion? Neben den jeweils jüngeren Kohorten vor allem die Kinder von Landwirten und qualifizierten Arbeitern und schließlich die Frauen.

In welchem Umfang haben sich hierbei Bildungsungleichheiten nach sozialer Herkunft und Geschlecht verändert? Wie am Beispiel für den Erwerb der Maturität gesehen, sind die herkunftsbedingten Ungleichheiten und Geschlechterunterschiede beim Zugang und Erwerb von Bildung in der Kohortenabfolge bis 1990 deutlich zurückgegangen. Aber für das Jahr 2000 hat sich wieder eine ebenso deutliche Zunahme der Bildungsungleichheiten im Vergleich zum Jahr 1990 ergeben. Die Zunahme ist für die soziale Herkunft ausgeprägter als für das Geschlecht. Dass die geschlechtstypischen Unterschiede im Bildungssystem unabhängig von der Entwicklung nach sozialer Herkunft verlief, konnte nunmehr auch für die Schweiz empirisch belegt werden. Diese Entwicklungen wurden anhand eines strukturell-individualistischen Erklärungsansatzes empirisch zutreffend vorhergesagt, während sich der konflikttheoretische Ansatz von Bourdieu und Passeron (1971) allenfalls bedingt bewährt hat. Hingegen erwies sich die Modernisierungstheorie ebenso wenig erklärungskräftig wie die MMI- oder EMI-These. Ob diese Entwicklung der Bildungsungleichheiten in der Schweiz historisch einmalig ist, wird sich mit der VZ 2010 zeigen, wenn die Daten für wissenschaftliche Analysen zur Verfügung stehen. 
Was sagen unsere Befunde für die Schweiz zur These dauerhafter Ungleichheiten im Bildungssystem? Im Sinne der strukturell-individualistischen Theorie von Boudon (1974) oder des konflikttheoretischen Ansatzes nach Bourdieu und Passeron (1971) sind trotz Bildungsexpansion und abnehmender sozialer Ungleichheit von Bildungschancen immer noch deutliche soziale Disparitäten beim Bildungszugang und Erwerb von Bildungsabschlüssen zu beobachten. Aber sie sind eben nicht dauerhaft, sondern verändern sich, offensichtlich in Abhängigkeit von den Rahmenbedingungen des Bildungssystems, im Zeitverlauf. Aber sie können, wie jüngst von Jann und Combet (2012) am Beispiel der intergenerationalen Mobilität in der Schweiz demonstriert, auch eine Folge der sozialen Ungleichheiten außerhalb des Bildungssystems und ihres Wandels sein (vgl. Solga 2012).

War hinsichtlich der Chancengleichheit die Bildungsexpansion in der Schweiz folgenlos geblieben? Entgegen mancherlei Hoffnung ist die Bildungsexpansion alleine ein stumpfes Instrument für mehr Chancengleichheit im Bildungssystem (vgl. Breen 2010). Vor allem, wenn, wie in der Schweiz, die sozialen Ungleichheiten außerhalb des Bildungssystems sehr groß sind (vgl. Stamm et al. 2003). Die relativ hohe sowie stabile ökonomische Ungleichheit in der Schweiz, auch bei einem stetigen berufsstrukturellen Wandel hin zur Tertiarisierung der Berufe mit hohen Anforderungen an die Ausbildung der Beschäftigten (vgl. Sheldon 2005), könnte für einen langen Zeitraum betrachtet den relativ langsamen Rückgang der herkunftstypischen Bildungsungleichheiten zumindest teilweise erklären. Vor allem hat sich die ökonomische Situation für Arbeiter, Landwirte und einfache Angestellte nach dem Zweiten Weltkrieg deutlich verbessert. So stiegen in der Schweiz nach 1945 die durchschnittlichen Realeinkommen bis Mitte der 1990er Jahre deutlich an, ohne dass sich die Einkommensspreizungen verändert hätten. Sie sind seitdem konstant. Der allgemeine ökonomische Wohlstand für Familien verbesserte sich bei einer ungleichen Verteilung in den 1970er Jahren und ist seit den 1980er Jahren ebenfalls konstant (vgl. Dell et al. 2005). Ob die sich verschärfende soziale Ungleichheit infolge der intergenerationalen Mobilität zu größeren Ungleichheiten im Schweizer Bildungssystem geführt hat, bedarf einer weiterführenden detaillierten Untersuchung.

Ebenso sollten die Folgen der in den 1990er Jahren eingeführten Berufsmaturität und die Institutionalisierung von Fachhochschulen auf die Entwicklung von herkunftsbedingten und geschlechtstypischen Bildungschancen in der Schweiz im 21. Jahrhundert untersucht werden. So ist es angesichts der hier angestellten theoretischen Überlegungen und vorgelegten Evidenz wahrscheinlich, dass diese institutionellen Veränderungen die „Ablenkung“ von Bildungsaufsteigern vom „Königsweg“ zu den Universitäten noch weiter verstärkt hat (vgl. für Effekte in Deutschland: Schindler und Reimer 2011). Aus konflikttheoretischer Sicht kann gemutmaßt werden, dass mit den Reformen der in allen Bevölkerungsschichten gestiegenen Aspiration für das Gymnasium und die Hochschulbildung entgegnet wurde, um die sozialen Aufsteiger auf diese neuen, weniger aufwendig und riskant erscheinenden Bildungswege zu lenken und dadurch die soziale Exklusivität der gymnasialen Maturität und des Universitätsstudiums zu erhalten (vgl. Weber 1972, S. 577). Entgegen der Behauptung, dass dadurch die Durchlässigkeit des höheren Bildungssystems zugunsten der sozial benachteiligten Gruppen erhöht wird, könnte gerade die zeitgleiche Einführung der Berufsmaturität und der Fachhochschulen zum Wiederanstieg der Bildungsungleichheit in den 1990er Jahren beigetragen haben. Zu überprüfen wäre dann mit aktuelleren Daten, ob diese Bildungsreformen in diesen Jahren auch nach 
der Jahrtausendwende zur sozialen Ungleichheit beim Erwerb der Studienberechtigung und des Hochschulzugangs beigetragen haben. In diesem Zusammenhang ist es dann ebenso interessant, die Frage zu untersuchen, ob nicht auch die „Bologna-Reform“ mit der Einführung eines zweistufigen Studiums an Universitäten und Fachhochschulen und eines neuen Hochschulabschlusses (Bachelor) die soziale Ungleichheit im Bildungssystem, nicht trotz, sondern gerade wegen der Bildungsexpansion und der Universalisierung weiterführender Bildung, verschärft hat (vgl. Powell et al. 2012).

\section{Literatur}

Baumert, Jürgen, und Gundel Schümer. 2001. Familiäre Lebensverhältnisse, Bildungsbeteiligung und Kompetenzerwerb. In PISA 2000: Basiskompetenzen von Schülerinnen und Schülern im internationalen Vergleich, Hrsg. Deutsches PISA-Konsortium, 323-407. Opladen: Leske + Budrich.

Beck, Michael, Franziska Jäpel und Rolf Becker. 2010. Determinanten des Bildungserfolgs von Migranten im Schweizer Bildungssystem. In Bildungsverlierer - Neue Ungleichheiten, Hrsg. Gudrun Quenzel und Klaus Hurrelmann, 313-337. Wiesbaden: VS Verlag für Sozialwissenschaften.

Becker, Rolf. 2003. Educational expansion and persistent inequalities of education: Utilizing the subjective expected utility theory to explain the increasing participation rates in upper secondary school in the Federal Republic of Germany. European Sociological Review 19:1-24.

Becker, Rolf. 2009. Wie können „bildungsferne“ Gruppen für ein Hochschulstudium gewonnen werden? Eine empirische Simulation mit Implikationen für die Steuerung des Bildungswesens. Kölner Zeitschrift für Soziologie und Sozialpsychologie 61:563-593.

Becker, Rolf. 2010. Soziale Ungleichheit im Schweizer Bildungssystem und was man dagegen tun könnte. In Schulübergang und Selektion - Forschungserträge und Umsetzungsstrategien, Hrsg. Markus Neuenschwander und Hans-Ueli Grunder, 91-108. Chur: Rüegger.

Becker, Rolf. 2012. Der Übergang ins Hochschulstudium: Prozesse und Mechanismen am Beispiel der deutschen Schweiz. In Bildung - Arbeit-Erwachsenwerden. Ein interdisziplinärer Blick auf die Transition im Jugend und jungen Erwachsenenalter, Hrsg. Max Bergman, Sandra Hupka-Brunner, Thomas Meyer und Robin Samuel, 305-331. Wiesbaden: Springer VS.

Becker, Rolf, und Anna Etta Hecken. 2009a. Higher education or vocational training? An empirical test of the rational action model of educational choices suggested by Breen and Goldthorpe (1997) and Esser (1999). Acta Sociologica 52:25-45.

Becker, Rolf, und Anna Etta Hecken. 2009b. Why are working-class children diverted from universities? European Sociological Review 25:233-250.

Becker, Rolf, und Walter Müller. 2011. Bildungsungleichheiten nach Geschlecht und Herkunft im Wandel. In Geschlechtsspezifische Bildungsungleichheiten, Hrsg. Andreas Hadjar, 55-75. Wiesbaden: VS Verlag für Sozialwissenschaften.

Becker, Rolf, Sigrid Haunberger und Frank Schubert. 2010. Studienfachwahl als Spezialfall der Ausbildungsentscheidung und Berufswahl. Zeitschrift für Arbeitsmarktforschung 42:292-310.

Best, Henning, und Christof Wolf. 2012. Modellvergleich und Ergebnisinterpretation in Logit- und Probit-Regressionen. Kölner Zeitschrift für Soziologie und Sozialpsychologie 64:377-395.

Blossfeld, Hans-Peter. 1988. Bildungsverläufe im historischen Wandel. Eine Längsschnittanalyse über die Veränderung der Bildungsbeteiligung im Lebenslauf dreier Geburtskohorten. In Bildung, Beruf, Arbeitsmarkt, Hrsg. Hans-Joachim Bodenhöfer, 259-302. Berlin: Duncker \& Humblot. 
Blossfeld, Hans-Peter, und Yossi Shavit. 1993. Dauerhafte Ungleichheiten. Zur Veränderung des Einflusses der sozialen Herkunft auf die Bildungschancen in dreizehn industrialisierten Ländern. Zeitschrift für Pädagogik 39:25-52.

Bornschier, Volker, und Doris Aebi. 1992. Rolle und Expansion der Bildung in der modernen Gesellschaft. Von der Pflichtschule bis zur Weiterbildung. Schweizerische Zeitschrift für Soziologie 18:539-567.

Boudon, Raymond. 1974. Education, opportunity, and social inequality. New York: Wiley.

Bourdieu, Pierre, und Jean-Claude Passeron. 1971. Die Illusion der Chancengleichheit. Untersuchungen zur Soziologie des Bildungswesens am Beispiel Frankreichs. Stuttgart: Klett.

Breen, Richard. 2010. Educational expansion and social mobility in the 20th century. Social Forces 89:365-388.

Breen, Richard, und John H. Goldthorpe. 1997. Explaining educational differentials. Towards a formal rational action theory. Rationality \& Society 9:275-305.

Breen, Richard, Ruud Luijkx, Walter Müller und Reinhard Pollak. 2009. Nonpersistent inequality in educational attainment: Evidence from eight European countries. American Journal of Sociology 114:1475-1152.

Breen, Richard, Ruud Luijkx, Walter Müller und Reinhard Pollak. 2010. Long-term trends in educational inequality in Europe: Class inequalities and gender differences. European Sociological Review 26:31-48.

Breen, Richard, Ruud Luijkx, Walter Müller und Reinhard Pollak. 2012. Bildungsdisparitäten nach sozialer Herkunft und Geschlecht im Wandel - Deutschland im internationalen Vergleich. In Soziologische Bildungsforschung. Sonderheft 52 der Kölner Zeitschrift für Soziologie und Sozialpsychologie, Hrsg. Rolf Becker und Heike Solga, 346-373. Wiesbaden: Springer VS.

Buchmann, Marlis, Stefan Sacchi, Markus Lamprecht und Hanspeter Stamm. 2007. Tertiary education expansion and social inequality in Switzerland. In Stratification in Higher Education, Hrsg. Yossi Shavit, Richard Arum und Adam Gomoran, 321-348. Stanford: Stanford University Press.

Buchmann, Marlis, und Maria Charles. 1993. The lifelong shadow: Social origins and educational opportunity in Switzerland. In Persistent inequality: Changing educational attainment in 13 countries, Hrsg. Yossi Shavit und Hans-Peter Blossfeld, 177-192. Boulder: Westview Press.

Busset, Thomas. 1993. Zur Geschichte der eidgenössischen Volkszählung. Neuchâtel: Bundesamt für Statistik.

Collins, Randall. 1979. The credential society. New York: Academic Press.

Dell, Fabian, Thomas Piketty und Emmanuel Saez. 2005. Income and wealth concentration in Switzerland over the 20th century. Discussion Paper No. 5090. London: Centre for Economic Policy Research.

Erikson, Robert, John H. Goldthorpe und Lucienne Portocarero. 1979. Intergenerational class mobility in three Western European societies: England, France, and Sweden. British Journal of Sociology 30:341-415.

Erikson, Robert, und Jan O. Jonsson. 1996. Explaining class inequality in education: The Swedish test case. In Can education be equalized? The Swedish case in comparative perspective, Hrsg. Robert Erikson und Jan O. Jonsson, 1-63. Boulder: Westview Press.

Erikson, Robert, und Frida Rudolphi. 2010. Change in social selection to upper secondary school primary and secondary effects in Sweden. European Sociological Review 26:291-305.

Flora, Peter. 1974. Die Bildungsentwicklung im Prozess der Staaten- und Nationenbildung. In Soziologie und Sozialgeschichte. Sonderheft 16 der Kölner Zeitschrift für Soziologie und Sozialpsychologie, Hrsg. Peter Christian Ludz, 194-319. Opladen: Westdeutscher Verlag.

Goldthorpe, John H. 1996. Class analysis and the reorientation of class theory: The case of persisting differentials in educational attainment. British Journal of Sociology 47:481-505. 
Hadjar, Andreas, und Joel Berger. 2010. Dauerhafte Bildungsungleichheiten in Westdeutschland, Ostdeutschland und der Schweiz: Eine Kohortenbetrachtung der Ungleichheitsdimensionen soziale Herkunft und Geschlecht. Zeitschrift für Soziologie 39:182-201.

Hega, Gunther M. 2000. Federalism, subsidiarity and education policy in Switzerland. Regional \& Federal Studies 10:1-35.

Heidenheimer, Arnold. 1997. Disparate ladders: Why school and university policies differ in Germany, Japan, and Switzerland. New Brunswick: Transaction Publishers.

Hinz, Thomas, und Karin Auspurg. 2011. Gruppenvergleiche bei Regressionen mit binären abhängigen Variablen - Probleme und Fehleinschätzungen am Beispiel von Bildungschancen im Kohortenverlauf. Zeitschrift für Soziologie 40:62-73.

Jann, Ben, und Benita Combet. 2012. Zur Entwicklung der intergenerationalen Mobilität in der Schweiz. Schweizerische Zeitschrift für Soziologie 38:177-199.

Lamprecht, Markus, und Hanspeter Stamm. 1996. Soziale Ungleichheit im Bildungswesen. Bern: Bundesamt für Statistik.

Lepsius, Rainer M. 1979. Soziale Ungleichheit und Klassenstrukturen in der Bundesrepublik Deutschland. In Klassen in der europäischen Sozialgeschichte, Hrsg. Hans-Ulrich Wehler, 166-209. Göttingen: Vandenhoeck \& Ruprecht.

Long, J. Scott. 1997. Regression models for categorical and limited dependent variables. Thousand Oaks: Sage.

Lucas, Samuel R. 2001. Effectively maintained inequality: Educational transitions and social background. American Journal of Sociology 106:1642-1690.

Mayer, Karl Ulrich, und Johannes Huinink. 1990. Alters-, Perioden- und Kohorteneffekte in der Analyse von Lebensverläufen oder: Lexis ade? In Lebensverläufe und sozialer Wandel. Sonderheft 31 der Kölner Zeitschrift für Soziologie und Sozialpsychologie, Hrsg. Karl Ulrich Mayer, 442-460. Opladen: Westdeutscher Verlag.

Meylan, Jean-Pierre. 1996. Die Erneuerung des Gymnasiums und die Anerkennung der Maturitäten. Stationen der Debatte 1968-1995. In Von der ,,Mittelschule von morgen “ zur Maturitätsreform 1995, Hrsg. EDK, 7-45. Bern: EDK.

Müller, Walter. 1998. Erwartete und unerwartete Folgen der Bildungsexpansion. In Die Diagnosefähigkeit der Soziologie. Sonderheft 38 der Kölner Zeitschrift für Soziologie und Sozialpsychologie, Hrsg. Jürgen Friedrichs, Rainer M. Lepsius und Karl Ulrich Mayer, 83-112. Opladen: Westdeutscher Verlag.

Müller, Walter, und Irena Kogan. 2010. Education. In Handbook of European societies, Hrsg. Stefan Immerfall und Göran Therborn, 217-289. New York: Springer.

Müller, Walter, und Markus Klein. 2008. Schein oder Sein: Bildungsdisparitäten in der europäischen Statistik Eine Illustration am Beispiel Deutschlands. Schmollers Jahrbuch 128:511-544.

Müller, Walter, Susanne Steinmann und Reinhart Schneider. 1997. Bildung in Europa. In Die westeuropäischen Gesellschaften, Hrsg. Stefan Hradil und Stefan Immerfall, 177-245. Opladen: Leske + Budrich.

Neugebauer, Martin, David Reimer, Steffen Schindler und Volker Stocké. 2013. Inequality in transitions to secondary school and tertiary education in the German school system. In Determined to succeed? Performance versus choice in educational attainment, Hrsg. Michelle Jackson, 56-88. Stanford: Stanford University Press.

Neugebauer, Martin, und Steffen Schindler. 2012. Early transitions and tertiary enrolment: The cumulative impact of primary and secondary effects on entering university in Germany. Acta Sociologica 55:19-36.

Pfeffer, Fabian T. 2008. Persistent inequality in educational attainment and its institutional context. European Sociological Review 24:543-565. 
Powell, Justin J. W., Nadine Bernhard und Lukas Graf. 2012. Amerikanisierung oder Europäisierung der (Aus-)Bildung? Die Bologna- und Kopenhagen-Prozesse und das neue europäische Modell der Hochschul- und Berufsbildung, In Soziologische Bildungsforschung. Sonderheft 52 der Kölner Zeitschrift für Soziologie und Sozialpsychologie, Hrsg. Rolf Becker und Heike Solga, 437-458. Wiesbaden: Springer VS.

Quast, Heiko, Heike Spangenberg, Bettina Hannover und Edith Braun. 2012. Der Einfluss von Studiengebühren auf die Studierbereitschaft. Zeitschrift für Erziehungswissenschaft 15:305-326.

Raftery, Adrian E., und Michael Hout. 1993. Maximally maintained inequality: Expansion, reform, and opportunity in Irish education, 1921-75. Sociology of Education 66:41-62.

Rieger, Andreas. 2001. Bildungsexpansion und ungleiche Bildungspartizipation am Beispiel der Mittelschulen im Kanton Zürich, 1830 bis 1980. Schweizerische Zeitschrift für Bildungswissenschaften 23:41-72.

Riphahn, Regina T., und George Sheldon. 2005. Arbeit in der alternden Gesellschaft: Der Arbeitsmarkt für ältere Menschen in der Schweiz. Basel: FAI.

Schindler, Steffen, und David Reimer. 2011. Differentiation and social selectivity in German higher education. Higher Education 61:261-275.

Schneider, Reinhart. 1982. Die Bildungsentwicklung in den westeuropäischen Staaten 1870-1975. Zeitschrift für Soziologie 11:207-226.

Schumann, Stephan. 2011. Leistungs- und Herkunftseffekte beim Hochschulzugang in der Schweiz. Zeitschrift für Pädagogik 57:246-268.

Sheldon, George. 2005. Der berufsstrukturelle Wandel der Beschäftigung in der Schweiz 19702000: Ausmaß, Ursachen und Folgen. Neuchâtel: Bundesamt für Statistik.

SKBF (Schweizerische Koordinationsstelle für Bildungsforschung). 2011. Swiss Education Report 2010. Aarau: Schweizerische Koordinationsstelle für Bildungsforschung.

Solga, Heike. 2012. Bildung und materielle Ungleichheiten. Der investive Sozialstaat auf dem Prüfstand. In Soziologische Bildungsforschung. Sonderheft 52 der Kölner Zeitschrift für Soziologie und Sozialpsychologie, Hrsg. Rolf Becker und Heike Solga, 459-487. Wiesbaden: Springer + VS.

Solga, Heike, und Rolf Becker. 2012. Soziologische Bildungsforschung - eine kritische Bestandsaufnahme. In Soziologische Bildungsforschung. Sonderheft 52 der Kölner Zeitschrift für Soziologie und Sozialpsychologie, Hrsg. Rolf Becker und Heike Solga, 7-43. Wiesbaden: Springer VS.

Stamm, Hanspeter, Markus Lamprecht und Rolf Nef. 2003. Soziale Ungleichheit in der Schweiz. Zürich: Seismo.

Stamm, Hanspeter, und Markus Lamprecht. 2005. Entwicklung der Sozialstruktur. Neuchâtel: Bundesamt für Statistik.

Treiman, Donald J. 1970. Industrialization and social stratification. Sociological Inquiry 40:207-234.

Turner, Ralph. 1960. Sponsored and contest mobility and the school system. American Sociological Review 25:855-867.

Weber, Max. 1972. Wirtschaft und Gesellschaft. Tübingen: Mohr.

Wettstein, Emil, und Philipp Gonon. 2009. Berufsbildung in der Schweiz. h.e.p Verlag: Bern.

Zahner, Claudia, et al. 2002. Für das Leben gerüstet? Die Grundkompetenzen der JugendlichenNationaler Bericht der Erhebung PISA 2000. Neuchâtel: Bundesamt für Statistik (BFS). 
Rolf Becker, 1960, Prof. Dr., Direktor der Abteilung für Bildungssoziologie an der Universität Bern. Forschungsgebiete: Bildungssoziologie, Sozialstrukturanalyse, Lebensverlaufsforschung, Methoden der empirischen Sozialforschung und angewandte Statistik, Rational-Choice-Theorien, Arbeitsmarkt- und Mobilitätsforschung, empirische Wahlforschung. Veröffentlichungen: Soziologische Bildungsforschung. Sonderheft 52 der Kölner Zeitschrift für Soziologie und Sozialpsychologie. Wiesbaden 2012 (hrsg. mit H. Solga). Diskriminierung durch Lehrpersonen oder herkunftsbedingte Nachteile von Migranten im Deutschschweizer Schulsystem? Schweizerische Zeitschrift für Soziologie 39, 2013 (mit M. Beck und F. Jäpel). Wie können „bildungsferne“ Gruppen für ein Hochschulstudium gewonnen werden? Eine empirische Simulation mit Implikationen für die Steuerung des Bildungswesens. Kölner Zeitschrift für Soziologie und Sozialpsychologie 4, 2009.

Christoph Zangger, 1986, Master of Arts in Sozialwissenschaften, Assistent, Abteilung Bildungssoziologie, Universität Bern. Forschungsgebiete: Bildungs- und Stadtsoziologie (Bildungskontexte), Sozialstrukturanalyse, Methoden der empirischen Sozialforschung. 